\title{
HiCanu: accurate assembly of segmental duplications, satellites, and allelic variants from high-fidelity long reads
}

\author{
Sergey Nurk, ${ }^{1,6}$ Brian P. Walenz, ${ }^{1,6}$ Arang Rhie, ${ }^{1}$ Mitchell R. Vollger, ${ }^{2}$ \\ Glennis A. Logsdon, ${ }^{2}$ Robert Grothe, ${ }^{3}$ Karen H. Miga, ${ }^{4}$ Evan E. Eichler, ${ }^{2,5}$ \\ Adam M. Phillippy, ${ }^{1}$ and Sergey Koren ${ }^{1}$ \\ ${ }^{1}$ Genome Informatics Section, Computational and Statistical Genomics Branch, National Human Genome Research Institute, \\ National Institutes of Health, Bethesda, Maryland 20894, USA; ${ }^{2}$ Department of Genome Sciences, University of Washington School \\ of Medicine, Seattle, Washington 98195, USA; ${ }^{3}$ Pacific Biosciences, Menlo Park, California 94025, USA; ${ }^{4}$ UC Santa Cruz Genomics \\ Institute, University of California, Santa Cruz, California 95064, USA; ${ }^{5}$ Howard Hughes Medical Institute, University of Washington, \\ Seattle, Washington 98195, USA
}

\begin{abstract}
Complete and accurate genome assemblies form the basis of most downstream genomic analyses and are of critical importance. Recent genome assembly projects have relied on a combination of noisy long-read sequencing and accurate shortread sequencing, with the former offering greater assembly continuity and the latter providing higher consensus accuracy. The recently introduced Pacific Biosciences (PacBio) HiFi sequencing technology bridges this divide by delivering long reads ( $>10 \mathrm{kbp}$ ) with high per-base accuracy (>99.9\%). Here we present HiCanu, a modification of the Canu assembler designed to leverage the full potential of HiFi reads via homopolymer compression, overlap-based error correction, and aggressive false overlap filtering. We benchmark HiCanu with a focus on the recovery of haplotype diversity, major histocompatibility complex (MHC) variants, satellite DNAs, and segmental duplications. For diploid human genomes sequenced to $30 \times \mathrm{HiFi}$ coverage, HiCanu achieved superior accuracy and allele recovery compared to the current state of the art. On the effectively haploid CHMI3 human cell line, HiCanu achieved an NG50 contig size of $77 \mathrm{Mbp}$ with a per-base consensus accuracy of 99.999\% (QV50), surpassing recent assemblies of high-coverage, ultralong Oxford Nanopore Technologies (ONT) reads in terms of both accuracy and continuity. This HiCanu assembly correctly resolves 337 out of 341 validation BACs sampled from known segmental duplications and provides the first preliminary assemblies of nine complete human centromeric regions. Although gaps and errors still remain within the most challenging regions of the genome, these results represent a significant advance toward the complete assembly of human genomes.
\end{abstract}

[Supplemental material is available for this article.]

Genome assembly is the process of reconstructing continuous genomic regions from shorter overlapping fragments, called reads (Miller et al. 2010; Nagarajan and Pop 2010). Recently, long-read sequencing technologies have significantly simplified assembly by generating multikilobase reads, which span most common genomic repeats (Chin et al. 2013; Koren et al. 2013; Koren and Phillippy 2014; Gordon et al. 2016; Bickhart et al. 2017; Kronenberg et al. 2018). Despite the per-base error rate of the input reads exceeding $10 \%$, state-of-the-art assembly methods are able to resolve instances of longer repeats with sequence divergence as low as $2 \%$ (Koren et al. 2017; Kolmogorov et al. 2019). However, a significant fraction of the human genome is represented by long segmental duplications (SDs) of higher sequence identity. According to the current annotation of the human reference (Bailey et al. 2001, 2002), 208 Mbp of sequence is contained within repeats $>20 \mathrm{kbp}$ with sequence identity $>98 \%$. Low accuracy of the long-read technologies has also made continuous reconstruc-

\footnotetext{
${ }^{6}$ These authors contributed equally to this work. Corresponding authors: adam.phillippy@nih.gov; sergey.koren@nih.gov

Article published online before print. Article, supplemental material, and publication date are at http://www.genome.org/cgi/doi/10.1101/gr.263566.120. Freely available online through the Genome Research Open Access option.
}

tion of individual haplotypes very challenging because humans can average less than one heterozygous variant per $1 \mathrm{kbp}$. Typical assembly strategies collapse the genome first and phase afterward by calling variants, partitioning the reads, and reassembling (Chin et al. 2016; Seo et al. 2016). State-of-the-art methods integrate different sequencing technologies (Chaisson et al. 2019; Kronenberg et al. 2019) or parental information (Koren et al. 2018) to obtain chromosome-scale, haplotype-resolved assemblies. However, these approaches have the downside of collapsing multicopy repeats in the assembly or not resolving alleles that differ at only a few positions.

Recently, Pacific Biosciences (PacBio) introduced a new data type, referred to as HiFi reads (Wenger et al. 2019). The process of generating HiFi reads involves DNA fragmentation, adapter ligation and fragment circularization, and multipass sequencing of the circularized fragments. The resulting signal is then computationally processed to obtain an accurate consensus sequence for each individual fragment. To ensure that each fragment undergoes sufficient sequencing passes to obtain a high consensus accuracy,

(C) 2020 Nurk et al. This article, published in Genome Research, is available under a Creative Commons License (Attribution 4.0 International), as described at http://creativecommons.org/licenses/by/4.0/. 
HiFi sequencing libraries are size selected for a target fragment size (currently up to $25 \mathrm{kbp}$ ).

Although the resulting read lengths are modest by the modern long-read sequencing standards-PacBio CLR reads frequently exceed $50 \mathrm{kbp}$, and ultralong Oxford Nanopore Technologies (ONT) reads can exceed even $100 \mathrm{kbp}$ (Jain et al. 2018b), HiFi is a major leap forward in terms of long-read read accuracy. As the accuracy of other long-read technologies have not exceeded 95\%, the median accuracy of current HiFi reads can exceed $99.9 \%$ (>Q30), making them a promising data type for separating highly similar repeat instances and alleles.

Early studies adopting HiFi sequencing showed improved variant calling and repeat resolution (Wenger et al. 2019; Vollger et al. 2020). However, these early assemblies were limited to resolving repeats with $>1 \%$ sequence divergence, owing to limitations of existing tools (Wenger et al. 2019). The recently developed Peregrine assembler (Chin and Khalak 2019) greatly reduced assembly runtime and improved consensus accuracy, removing the need for postprocessing, but did not address the issue of suboptimal repeat resolution or allele separation. Other recent work combined $\mathrm{HiFi}$ sequencing with complementary data types, such as parental information (Wenger et al. 2019), Hi-C (Garg et al. 2019), and Strand-seq (Porubsky et al. 2019), to obtain chromosome-scale, haplotype-resolved assemblies.

In the following sections, we present HiCanu, a modification of the Canu assembler (Koren et al. 2017) designed to take full advantage of the high accuracy of HiFi reads. We evaluate HiCanu's ability to resolve near-identical genomic repeats, with a focus on centromeric repeats and SDs by comparing our results to other HiFi and recent ultralong Oxford Nanopore-based human assemblies (Miga et al. 2020; Shafin et al. 2020). Furthermore, we evaluate HiCanu's ability to capture both alleles in large phase blocks in a diploid human genome. based on their overlaps to other reads to remove any chimeric sequences or sequencing adapters (see overlap based trimming in Koren et al. 2017), and the overlaps are recomputed on the trimmed reads. The overlap error adjustment (OEA) module (Holt et al. 2002; Koren et al. 2017) examines read overlap pileups to identify remaining sequencing errors in the individual reads and recomputes overlap alignment identities. Following our observation that microsatellite repeat arrays are also prone to HiFi read errors, the OEA procedure was modified to ignore any differences within these regions when computing the final alignment identity of two overlapping reads. Compared with the initial reads (Fig. 1A), homopolymer compression (Fig. 1B), pileup-based read correction (Fig. 1C), and ignoring differences in microsatellite repeats (Fig. 1D) result in a drastic reduction of observed sequencing errors. Draft contigs are then formed from the adjusted overlaps using Canu's Bogart module (Koren et al. 2017), modified to better handle heterozygous variants and consider only high-identity read overlaps. The final contig sequences are obtained by computing a consensus across the original, uncompressed reads, arranged according to the layout of their compressed versions. Similar to many modern assemblers, when faced with a diploid genome, HiCanu outputs contigs as "pseudohaplotypes" that preserve local allelic phasing but may switch between haplotypes across longer distances. A single set of contigs representing all resolved alleles is output regardless of ploidy, and additional processing with a tool such as Purge_dups (Guan et al. 2020) is required to partition the contigs into primary and alternate allele sets.

\section{Drosophila genome assembly}

We first evaluated HiCanu on a 24-kbp HiFi library from a Drosophila melanogaster F1 hybrid (ISO1×A4; see Data access). To match typical coverage, the HiFi data set was down-sampled to

\section{Results}

\section{HiCanu overview}

HiCanu builds on the original Canu pipeline, replacing or significantly modifying its core methods. Here we provide an overview of the new pipeline, highlighting the introduced changes, although a more detailed description of individual steps can be found in the Methods section. Whereas the original Canu pipeline starts with read self-correction, which can homogenize reads from different alleles or near-identical repeat instances, HiCanu begins by compressing all consecutive copies of the same nucleotide to a single base (e.g., "AA..." becomes "A"). In accordance with the earlier observation that misestimation of homopolymer length is the primary error mode of HiFi technology (Wenger et al. 2019), the resulting homopolymer-compressed reads (or "compressed reads" for short) accurately encode the transitions between different bases of the underlying genomic regions. The compressed reads are then trimmed

\section{A TCCCCAGT-CTTCCT--ACGTT-GAT CGTCCC-TGTTCTTCCTCTACGTTTG}

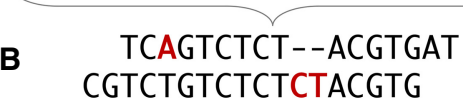
CGTCTGTCTCTCTACGTG
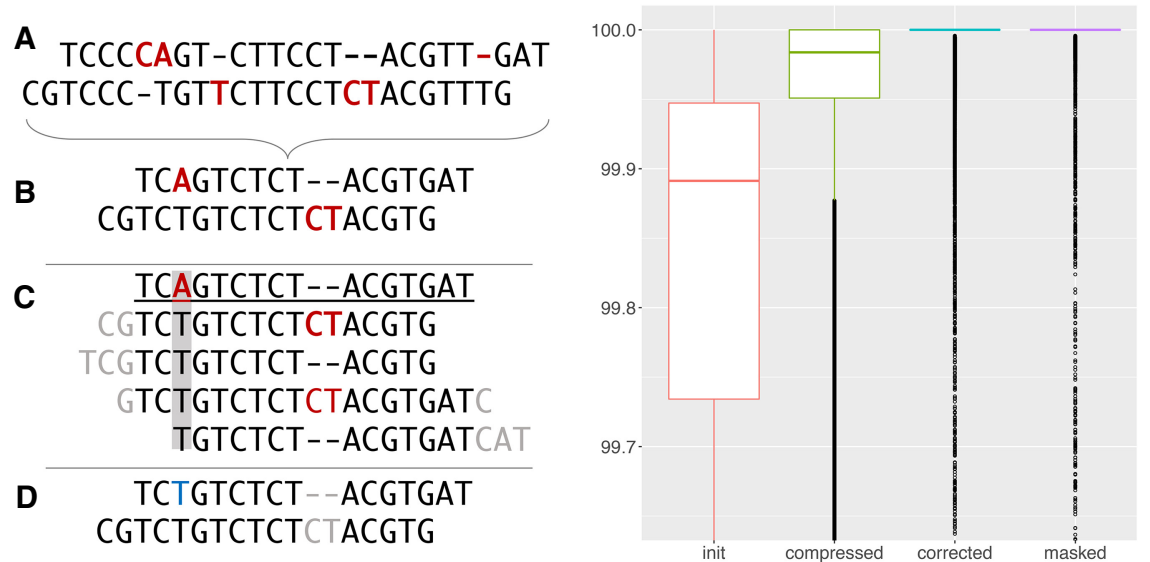

Figure 1. Impact of HiCanu processing on observed read quality. $(A)$ Two hypothetical reads are shown with sequencing errors highlighted in red. $(B)$ The first step of HiCanu is to compress homopolymers, which obscures homopolymer length errors but retains enough information to accurately distinguish reads from different genomic loci. (C) Overlaps are then computed for the compressed reads, and remaining errors are identified by examining the alignment pileups (gray rectangle). (D) Finally, after correcting the identified errors (blue) and ignoring indels in regions of known systematic error (gray), the resulting overlap is $100 \%$ identical. (Right) Sequence identity of reads from a 20 -kbp HiFi library measured against the CHM13 Chromosome X reference sequence v0.7 (Miga et al. 2020) after each step of HiCanu processing (Supplemental Note 1). Separate boxplots are shown for initial raw HiFi reads (init), homopolymer-compressed reads (compressed), OEA-corrected reads (corrected), and corrected reads after ignoring differences in microsatellite repeats (masked). The median read identity, indicated by solid segments, increases from $<99.9 \%$ to $100 \%$ (note the plot shows $y$-range of $99.65 \%-100 \%$ ). Supplemental Table S1 also shows how HiCanu processing increases the percentage of perfectly aligned ( $100 \%$ identity) HiFi reads from $<1 \%$ to $>97 \%$.

\section{Genome Research}

www.genome.org 
$40 \times$ and assembled with the HiFi-specific tools, HiCanu and Peregrine (Chin and Khalak 2019), as well as the conventional long-read assembler Canu. Canu was chosen as it was previously shown to achieve the highest assembly continuity and superior repeat resolution among other popular long-read assemblers on $\mathrm{HiFi}$ data (Wenger et al. 2019). For comparison, we also include a Canu assembly of $200 \times$ PacBio single-pass reads (CLR) for the same organism. Contigs $<50 \mathrm{kbp}$ were filtered from the assemblies in order to exclude low-quality sequences consisting of only a few reads.

Total assembly size varied between HiCanu (301 Mbp), Canu (293 Mbp), Peregrine (162 Mbp), and CLR (294 Mbp). Besides Peregrine, the assembly sizes were more than twice that of the 144-Mbp D. melanogaster haploid reference genome (Hoskins et al. 2015), suggesting that both haplotypes of the highly heterozygous F1 were successfully assembled (heterozygosity estimated at $0.7 \%$ by GenomeScope) (Supplemental Fig. S1; Vurture et al. 2017). The large fraction of duplicated BUSCO (Waterhouse et al. 2018) genes also supported the hypothesis that the assemblies captured alleles from both haplotypes (Supplemental Table S2). To facilitate like-for-like comparison of all assemblies, we ran Purge_dups (Guan et al. 2020) to identify alleles and divide the assemblies into primary and alternate contig sets (Methods). Assembly statistics were then computed for both contig sets and the results summarized in Table 1 . Per-base consensus accuracy was estimated using Merqury (Rhie et al. 2020) with Illumina sequencing data from the $D$. melanogaster F1 parental strains (Supplemental Table S2; Supplemental Note 2).

The primary contig sets across all assemblies reported high BUSCO completeness (>98\%). BUSCO duplication values were $<2 \%$ across all contig sets. The HiCanu primary contig set was noticeably more continuous than any other assembly as measured by NG50 (N such that half the haploid genome size is represented by contigs of this size or greater). Canu and HiCanu showed very similar per-base consensus accuracy, radically improving on both Peregrine and CLR assemblies. The Peregrine assembly collapsed both haplotypes together and output few alternate contigs (total length <21 Mbp). HiCanu improved over all other assemblies with respect to the total size, BUSCO completeness, and continuity of the alternate set (including a threefold improvement in NG50 over Canu).

To assess the integrity of the assemblies, we used QUAST v5.02 (Gurevich et al. 2013) to compare the assemblies against the chromosome arms of the D. melanogaster ISO1 reference. Because Purge_dups can split and/or trim the initial contigs but has a negligible effect on continuity, we report structural correctness of the original assemblies. Considering that one of the haplotypes (derived from the A4 parental strain) is expected to differ significantly from the reference, we adjusted QUAST's parameters to detect only large-scale genomic differences (Methods). Although the HiCanu assembly reported three more structural discrepancies than Canu (seven vs. four), it maintained the highest NG50 and alternate contig BUSCO completeness.

HiFi reads alone cannot be used to infer phasing across homozygous regions longer than the read length, so the contigs produced by HiCanu (and Canu) represent "pseudohaplotypes," which may switch between haplotypes. However, for highly heterozygous genomes with short regions of homozygosity, HiCanu is expected to produce a low number of haplotype switches and mostly preserve long-range phasing. We used Merqury (Rhie et al. 2020) to split the initial contigs into continuous phase blocks, based on haplotype-specific $k$-mer markers inferred from parental Illumina reads (Supplemental Note 2). As a baseline, we considered a haplotype-resolved assembly produced by TrioCanu (Koren et al. 2018) generated using a combination of CLR reads and parental Illumina data. The HiCanu primary (alternate) contig set has an estimated phase block NG50 of $7.62 \mathrm{Mbp}$ (4.45 Mbp), a maximal block length of $25.4 \mathrm{Mbp}$ (10.1 Mbp), and a low percentage of discordant markers within predicted haplotype blocks (switch rate) of $0.03 \%(0.02 \%)$. For comparison, the TrioCanu assembly has a paternal-ISO (maternal-A4) phase block NG50 of 13.9 Mbp (21.39 Mbp), a max block size of 24.7 Mbp (27.7 $\mathrm{Mbp})$, and an intra-block switch rate of $0.1 \%(0.04 \%)$. In contrast, the phase blocks of all other considered assemblies are much less continuous (at least a 3.5-fold drop in phase block NG50 compared to HiCanu) and, in the case of Peregrine and CLR assemblies, a much higher switch error (Table 1).

\section{Human genome assemblies}

We next ran HiCanu, Canu, and Peregrine on three different human data sets (see Data access): a 20-kbp library of the completely homozygous cell line CHM13 (Kronenberg et al. 2018; Miga et al. 2020; Vollger et al. 2020), a 15-kbp library of the Ashkenazic cell line HG002 from the Personal Genome Project (Church 2005; Wenger et al. 2019), and a combined library (12\% $10 \mathrm{kbp}, 62 \%$ $15 \mathrm{kbp}, 26 \% 20 \mathrm{kbp}$ ) for the Puerto Rican cell line HG00733 from the 1000 Genomes Project (The 1000 Genomes Project Consortium 2012; Porubsky et al. 2019). All data sets consist of

Table 1. D. melanogaster ISO1×A4 assembly benchmarking results for PacBio CLR and HiFi

\begin{tabular}{|c|c|c|c|c|c|c|c|}
\hline Assembly & $\begin{array}{l}\text { Size } \\
(\mathrm{Mbp})\end{array}$ & $\begin{array}{l}\text { NG50 } \\
\text { (Mbp) }\end{array}$ & $\begin{array}{l}\text { Quality } \\
\text { (QV) }\end{array}$ & $\begin{array}{l}\text { BUSCO } \\
\text { complete }\end{array}$ & $\begin{array}{l}\text { Phase block NG50 } \\
\text { (Mbp) }\end{array}$ & $\begin{array}{c}\text { Intra-block switch } \\
\text { error }\end{array}$ & $\begin{array}{c}\text { QUAST } \\
\text { (diffs per Mbp) }\end{array}$ \\
\hline Canu + Purge_dups & 141.81 & 14.09 & 37.4 & $98.5 \%$ & 0.42 & $3.86 \%$ & 0.018 \\
\hline CLR & 128.15 & 0.31 & 35.5 & $86.7 \%$ & 0.25 & $2.97 \%$ & \\
\hline Peregrine & 141.59 & 12.68 & 32.9 & $98.2 \%$ & 0.07 & $1.78 \%$ & 0.062 \\
\hline $\mathrm{HiFi}^{-}$ & 20.53 & 0.00 & 33.5 & $1.0 \%$ & 0.00 & $3.71 \%$ & \\
\hline Canu + Purge_dups & 145.19 & 13.72 & 51.9 & $98.7 \%$ & 2.04 & $0.03 \%$ & 0.015 \\
\hline $\mathrm{HiFi}$ & 130.23 & 1.28 & 46.9 & $93.7 \%$ & 1.26 & $0.03 \%$ & \\
\hline HiCanu + Purge_dups & 146.27 & 20.16 & 51.0 & $98.8 \%$ & 7.62 & $0.03 \%$ & 0.025 \\
\hline $\mathrm{HiFi}$ & 132.53 & 4.54 & 46.7 & $95.5 \%$ & 4.45 & $0.02 \%$ & \\
\hline
\end{tabular}

A genome size of $143,726,002$ was used for NG50 computation. Only contigs $\geq 50$ kbp were used for all analyses except QUAST. Each assembly is reported after running Purge_dups, with the primary reported on top and alts on the bottom of each row. Phred-style consensus quality values are $\mathrm{QV}=$ $-10 \log P($ error); for example, QV40 $=99.99 \%$ accuracy. QUAST diffs reports the number of large structural discrepancies observed between the assemblies before Purge_dups (and therefore have only one entry per assembly) and the D. melanogaster ISO1 reference genome normalized by the assembly size (in Mbp). The best performing result for each genome in each metric is highlighted in bold. 
approximately $30 \times \mathrm{HiFi}$ sequencing coverage. For the HG002 data set, we reused the best assembly from a recent study (Wenger et al. 2019) as it reflects a Canu 1.7.1 assembly before HiCanu's development and the associated improvements to Canu's core modules. We additionally included the most continuous published (pseudohaplotype) assemblies of the same genomes, which relied on ultralong Oxford Nanopore reads to achieve state-of-the-art repeat resolution (Miga et al. 2020; Shafin et al. 2020). As before, contigs $<50 \mathrm{kbp}$ were excluded from analysis. As the sizes of HiCanu assemblies for the diploid data sets HG002 and HG00733 were $5.30 \mathrm{Gbp}$ and $5.46 \mathrm{Gbp}$, respectively, compared with a haploid genome size of $3.1 \mathrm{Gbp}$, we again postprocessed all diploid assemblies with Purge_dups and computed statistics for both primary and alternate contig sets.

Per-base consensus quality was again estimated by Merqury (Rhie et al. 2020) using Illumina data from the corresponding genome (Supplemental Note 2; Supplemental Table S3). To assess the structural correctness of the assemblies, we followed the methodology of Shafin et al. (2020). Namely, structural differences reported by QUAST v5.0.2 versus the human reference genome GRCh38 (Schneider et al. 2017) were postprocessed to ignore breakpoints in centromeric regions and annotated SDs, in order to reduce the number of false positives (Methods; Supplemental Table S3). As before, because Purge_dups may introduce or correct misassemblies as it modifies the contigs, the structural correctness assessment was performed on the original assemblies.

Primary contig summary statistics for the three human genomes are presented in Table 2 . The continuity of HiCanu assemblies, as measured by NG50, exceeded that of all other HiFi-based assemblies and even rivaled the continuity of Nanopore ultralong-read assemblies. Reported rates of structural differences for HiCanu was on par with the other assemblies. For consensus accuracy, the HiCanu primary contig sets exceeded QV50 (99.999\% ac- curacy) and alternate contigs sets exceeded QV40 (99.99\% accuracy), whereas the unpolished Nanopore assemblies failed to exceed QV30 (99.9\%). Although Nanopore assemblies currently require polishing with complementary technologies to maximize consensus accuracy, we discourage polishing HiCanu HiFi assemblies, because the available polishing pipelines may map reads back to the wrong repeat copies and actually introduce errors during polishing.

The total length of the HiCanu alternate contig sets exceeded 2 Gbp, highlighting its ability to separate human alleles (corresponding values across other assemblies did not exceed 400 Mbp). The following section, "Human haplotype phasing," further explores allele separation and phasing across these assemblies. The drastic improvements in consensus accuracy and allele separation for Canu versus HiCanu assemblies of HGOO2 is likely owing to Canu improvements and bug fixes made during the HiCanu development process, whereas the CHM13 and HG00733 assemblies represent the latest Canu version and the differences are less pronounced.

For CHM13 and HG00733 genomes, we additionally validated the assemblies against long continuous fragments of the corresponding genome, earlier reconstructed via bacterial artificial chromosome (BAC) sequencing (see Data access; no BACs were available for HG002). Many of these so-called "challenge" BACs were deliberately selected from genomic regions that pose significant assembly challenges (i.e., regions with SDs), making them useful for assembly benchmarking (Chin and Khalak 2019; Miga et al. 2020; Shafin et al. 2020; Vollger et al. 2020). Table 3 summarizes how well the challenge BACs are captured by different assemblies. To recognize a BAC as "resolved" within the assembly, we required $99.5 \%$ of the BAC length to be aligned to a single contig by minimap2 (Methods; Li 2018). Assembly sequence accuracy was measured as the median alignment identity of resolved

Table 2. Human assembly benchmarking results for ultralong Oxford Nanopore and PacBio HiFi

\begin{tabular}{|c|c|c|c|c|c|}
\hline Genome & Assembly & Size (Gbp) & NG50 (Mbp) & Quality (QV) & QUAST (diffs per Gbp) \\
\hline \multirow[t]{4}{*}{ CHM13 } & $\mathrm{ONT}^{\mathrm{a}}$ & 2.98 & 74.06 & 28.9 & 35.2 \\
\hline & Peregrine & 2.87 & 37.30 & 50.7 & 40.8 \\
\hline & Canu & 3.03 & 45.63 & 61.7 & 40.6 \\
\hline & HiCanu & 3.05 & 77.12 & 58.1 & 45.9 \\
\hline \multirow{8}{*}{ HG002 } & $\mathrm{ONT}^{\mathrm{b}}$ & 2.83 & 32.34 & 23.2 & 59.0 \\
\hline & & 0.07 & 0.00 & 23.4 & \\
\hline & Peregrine & 2.82 & 32.09 & 46.0 & 35.7 \\
\hline & & 0.04 & 0.00 & 33.5 & \\
\hline & Canu $^{c}$ & 2.92 & 26.67 & 33.7 & 51.4 \\
\hline & & 0.12 & 0.00 & 43.2 & \\
\hline & HiCanu & 2.99 & 46.39 & 51.8 & 44.6 \\
\hline & & 2.15 & 0.18 & 45.1 & \\
\hline \multirow[t]{8}{*}{ HG00733 } & $\mathrm{ONT}^{\mathrm{b}}$ & 2.83 & 40.55 & 23.9 & 68.5 \\
\hline & & 0.06 & 0.00 & 23.8 & \\
\hline & Peregrine & 2.81 & 31.53 & 46.3 & 75.4 \\
\hline & & 0.04 & 0.00 & 34.2 & \\
\hline & Canu & 2.95 & 23.00 & 50.5 & 76.1 \\
\hline & & 0.36 & 0.00 & 41.5 & \\
\hline & HiCanu & 2.97 & 38.43 & 50.6 & 57.5 \\
\hline & & 2.32 & 0.23 & 43.3 & \\
\hline
\end{tabular}

A genome size of $3,098,794,149$ was used for computing NG statistics. As in Table 1, only contigs $\geq 50$ kbp were used for all analyses except QUAST. Each assembly is reported after running Purge_dups, with the primary reported on top and alts on the bottom of each row. Structural differences in centromeric regions and SDs were ignored owing to instability in these regions, and diffs were normalized by assembly size (in Gbp). Supplemental

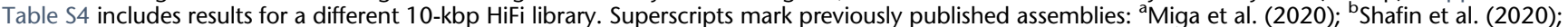
'Wenger et al. (2019). All ONT assemblies were generated by Canu, as it achieved the highest NG50 and BAC resolution. The best-performing result for each genome in each metric is highlighted in bold. Statistics for assemblies from Garg et al. (2019) and Porubsky et al. (2019) are included in Supplemental Tables S3 and S5.

\section{Genome Research}

www.genome.org 
Table 3. "Challenge" BAC validation of human assemblies

\begin{tabular}{llcr}
\hline Genome & Assembly & No. of BACs resolved & Median QV \\
\hline CHM13 & ONT & $314 / 341$ & 23.3 \\
& Peregrine & $136 / 341$ & 37.3 \\
& Canu & $308 / 341$ & 40.6 \\
HG00733 & HiCanu & $\mathbf{3 2 6 / 3 4 1}$ & $\mathbf{4 0 . 7}$ \\
& ONT & $124 / 179$ & 18.9 \\
& Peregrine & $74 / 179$ & 27.0 \\
& Canu & $122 / 179$ & 28.2 \\
\hline
\end{tabular}

The criteria for considering a BAC "resolved" is described in the main text and Methods. The alignment identity of each resolved BAC was computed individually and the median of these values reported as a Phred-style quality value. No validation BACs were available for HG002. Superscripts mark previously published assemblies: ${ }^{a}$ Miga et al. (2020); ${ }^{b}$ Shafin et al. (2020). All ONT assemblies were generated by Canu, as it achieved the highest NG50 and BAC resolution. The best-performing result for each genome in each metric is highlighted in bold. Statistics for assemblies from Garg et al. (2019) and Porubsky et al. (2019) are included in Supplemental Table S5.

BACs. Note that HiCanu resolved the highest number of BACs across all considered assemblies and also achieved the highest BAC alignment quality (Table 3; Supplemental Tables S4, S5).

A deeper investigation of the unresolved CHM13 BAC sequences indicated that 11 BACs likely contain assembly errors or cloning artifacts themselves (Supplemental Note 3; Supplemental Figs. S2-S5). Manual inspection of HiFi read alignments did not reveal any standard misassembly signatures in the corresponding regions of the HiCanu assembly, providing evidence that HiCanu was correct in these cases and able to resolve 337 out of 341 (99\%) of the CHM13 challenge BACs (Supplemental Table S6).

Although the challenge BACs are useful for validation, they do not represent the full landscape of human repeats. To further assess the ability of HiFi reads and different assemblers to resolve genomic repeats, we used the method of Vollger et al. (2019) to identify collapsed repeat instances in the CHM13 assemblies. We identified $\sim 21.7 \mathrm{Mbp}$ of collapsed repeats corresponding to at least $56 \mathrm{Mbp}$ of unresolved repetitive sequence. The HiCanu assembly had the lowest number of bases in regions identified as collapsed repeats, as well as the smallest amount of repetitive sequence predicted to be missing from the assembly (Supplemental Table S7; Supplemental Fig. S6). A complementary mapping-based analysis confirmed the comparatively high completeness of the HiCanu assembly and classified the majority (80\%) of missing sequence as satellite repeats, suggesting good recovery of all other human repeat classes (Supplemental Fig. S6).

\section{Human haplotype phasing}

When assembling a diploid genome, an assembler must choose to either collapse alleles into a single sequence or preserve them as two separate sequences. Collapsing heterozygosity results in a mosaic consensus that may not faithfully represent any allele and can introduce frameshifting errors within coding sequence.

HiCanu assemblies of the diploid human genomes included $>2$ Gbp of alternate contigs, with high BUSCO completeness for both primary and alternate contig sets $(>94 \%$ and $>75 \%$, respectively) (Supplemental Table S8). We again used Merqury (Rhie et al. 2020) to analyze the phase blocks using parental Illumina data (Supplemental Note 2). The phase block NG50s of HiCanu primary $(0.6 \mathrm{Mbp})$ and alternate $(0.1 \mathrm{Mbp})$ contig sets were the highest across all considered assemblies (2.5-fold higher than next best) (Supplemental Table S8). Note that the human phase block NG50s are significantly shorter than for the D. melanogaster F1 hybrid but are longer than a typical human gene. For compari- son, Supplemental Table S8 also includes statistics for the recently obtained haplotype-resolved assemblies of HG002 (Garg et al. 2019) and HG00733 (Porubsky et al. 2019). These recent studies have shown that multimegabase NG50 phase blocks can be obtained by integrating HiFi data with long-range linking information derived from Hi-C (Garg et al. 2019) or Strand-seq data (Porubsky et al. 2019).

To assess the phasing accuracy, we used a gold-standard variant set from the Genome in a Bottle (GIAB) consortium (Supplemental Table S9; Zook et al. 2019). HiCanu has an $F_{1}$ score of $95.37 \%$, which is $>50 \%$ higher than the next best HiFi assembly and similar to another assembly combining both HiFi and Hi-C data (97.7\%) (Garg et al. 2019). Finally, we validated the recovery of complex, clinically relevant alleles; we compared assembly typing results for the six classical human leukocyte antigen (HLA) genes (Dilthey et al. 2019) to the known alleles for HG002 and HG00733, obtained by previous studies (Supplemental Table S10; Chin et al. 2019; Shafin et al. 2020). Only HiCanu and TrioCanu were able to recover all alleles with 100\% sequence identity (Supplemental Tables S10, S11). The HiCanu contigs expectedly switch between the haplotypes, but there is only one switch in the MHC region. The Hi-C-phased HG002 assembly from Garg et al. (2019) is phased across the length of the MHC region but contains consensus errors (e.g., both HLA-DRB1 alleles). The Strandseq-phased HG00733 assembly from Porubsky et al. (2019) is also phased across the length of the MHC region but incorrectly represents HLA-A and HLA-B as homozygous (with both alleles in the assembly matching one ground-truth allele). Both the Garg et al. (2019) and Porubsky et al. (2019) methods rely on initially collapsed assemblies that are then phased using the longrange data. These results suggest that separation of haplotypes early in the assembly process (rather than trying to recover them from collapsed assemblies) may improve the accurate recovery of heterozygous variation.

\section{Complex regions of the $\mathrm{CHMl3}$ human genome}

The CHM13 HiCanu assembly (Tables 2, 3; Fig. 2) exceeded the predictions of our prior model of human assembly continuity (Supplemental Note 5; Supplemental Fig. S7). To validate this result, we focused on the performance of HiCanu within some of the most difficult-to-assemble regions of the genome, namely, centromeres and SDs. Unlike past assemblies of the human genome, including clone-based assemblies, HiCanu generated several contigs spanning mega-bases of satellite DNA. The CHM13 


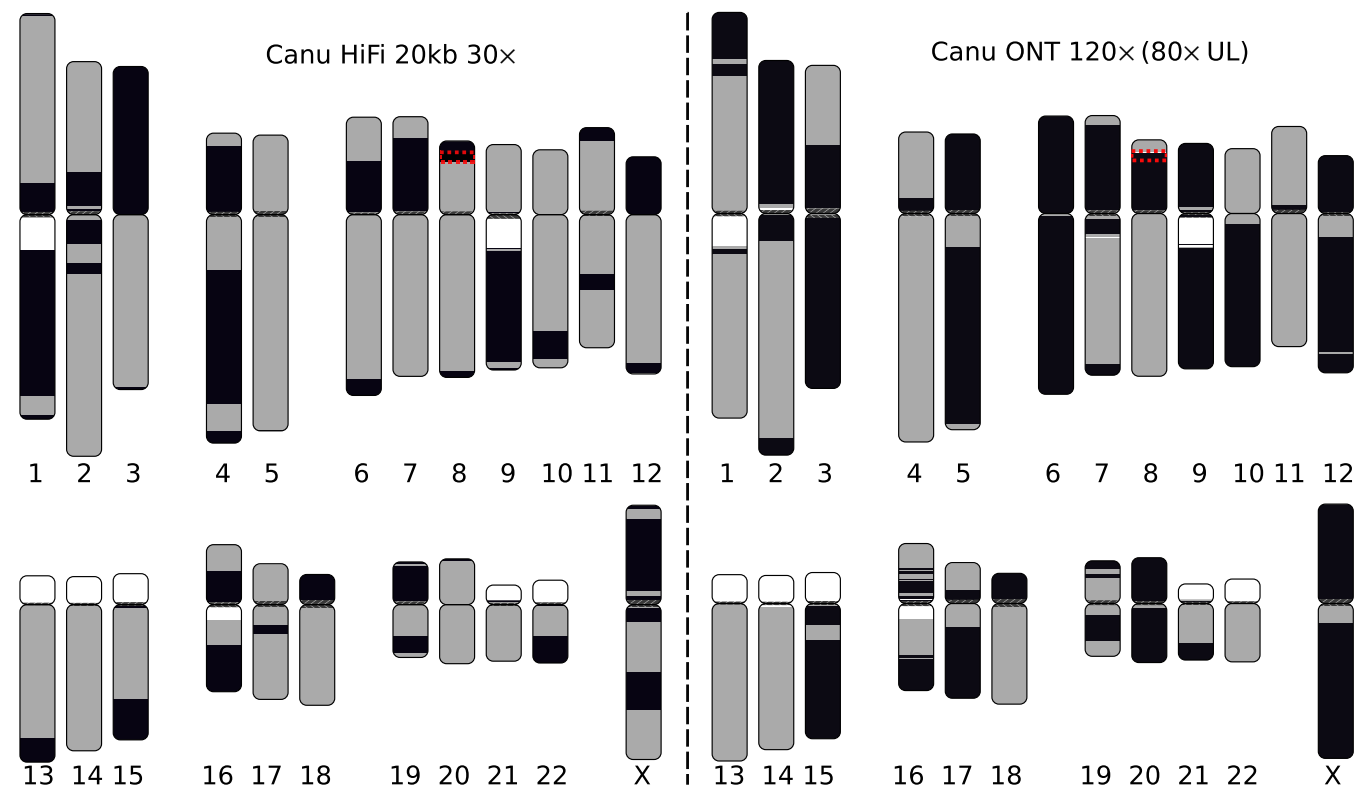

Figure 2. Visual representation of the most continuous HiFi-based and Nanopore-based assemblies of the CHM13 genome. HiCanu assembly of the 20$\mathrm{kbp} \mathrm{HiFi} \mathrm{data} \mathrm{set} \mathrm{(left)} \mathrm{and} \mathrm{Canu} \mathrm{assembly} \mathrm{of} \mathrm{an} \mathrm{ultralong} \mathrm{Nanopore} \mathrm{data} \mathrm{set} \mathrm{(right).} \mathrm{White} \mathrm{regions} \mathrm{indicate} \mathrm{gaps} \mathrm{in} \mathrm{the} \mathrm{current} \mathrm{reference} \mathrm{genome,} \mathrm{and} \mathrm{each}$ gray and black block indicates a continuous contig alignment. Color switches from gray to black represent either the end of a contig or an alignment break. Assemblies were aligned to GRCh38 using MashMap (Jain et al. 2018a), and plots were generated using coloredChromosomes (Böhringer et al. 2002) as previously described (Berlin et al. 2015; Jain et al. 2018b). Note that some chromosomes (e.g., Chr X) are better resolved by the Nanopore assembly owing to the presence of near-perfect repeats. At the same time, chromosomes containing more diverged repeats (e.g., Chr 7 and Chr 16) are better resolved by the HiFi assembly. We note that some gaps in the HiFi assembly are caused by sequence-specific biases of current HiFi sequencing protocols (Supplemental Note 4). The red box highlights the defensin beta gene family on Chromosome 8p23.1 which is split in both assemblies and detailed in Figure 4 .

HiCanu assembly contains nine of 23 (39\%) expected centromere regions: Chromosomes 2, 3, 7, 8, 10, 12, 16, 19, and 20 (Supplemental Note 6; Supplemental Table S11). The structure of these regions was consistent with an expectation of one or more higher-order repeat (HOR) array(s) flanked by more divergent tracts of monomeric satellite DNA (Willard and Waye 1987; Schueler et al. 2001; She et al. 2004). Mapped read depth of both HiFi and ultralong Oxford Nanopore data (Miga et al. 2020) across these contigs shows relatively uniform sequence coverage that spans the $\alpha$-satellite HOR array(s) into the unique sequences on the p- and q-arms (Fig. 3; Supplemental Note 6; Supplemental Fig. S8). The structure and length of the centromeric HOR array (s) in each contig is highly concordant with those reported in the literature (for review, see McNulty and Sullivan 2018).

It is noteworthy that HiCanu generated a draft assembly of the CHM13 Chromosome 19 centromere (Fig. 3). Historically, this region has been considered to be one of the more challenging centromeres to reconstruct because it carries multiple HOR tracts and shares $\alpha$-satellite sequences with the centromere regions from Chromosomes 1 and 5 (Hulsebos et al. 1988; Baldini et al. 1989; Pironon et al. 2010; Sullivan et al. 2017; McNulty and Sullivan 2018). HiCanu was not only able to assemble a contig that completely spans this centromere but also accurately distinguished three distinct HOR tracts (D19Z1, D19Z2, and D19Z3) (Supplemental Note 6; Supplemental Fig. S9). This contig revealed a more complete representation of the HOR structure of the D19Z1 HOR unit (13-mer vs. 10-mer) (Supplemental Figs. S9A, S10; Hulsebos et al. 1988; Puechberty et al. 1999), a Chromosome 19specific dimeric HOR (D19Z3) (Supplemental Figs. S9B, S10; Baldini et al. 1989; Finelli et al. 1996), and two complex HORs (expected to represent D19Z2) (Supplemental Note 6; Supplemental
Fig. S10). Alignment of HiFi sequence data to the corresponding HiCanu contig did not reveal any coverage anomalies (e.g., large dips or spikes) that could indicate the presence of structural errors. However, marker-assisted alignment of ultralong Oxford Nanopore data (Miga et al. 2020), an orthogonal data set, showed a coverage drop coinciding with the D19Z1 array. This may indicate a misassembly, mismapping of the noisy sequencing data, or biases in sequencing coverage. Because of the lack of a validated truth set in such regions, this will require extensive wet-laboratory validation and is left for future work.

Beyond the obvious challenge of centromere assembly, SDs represent another significant impediment and have been estimated to account for $68 \%$ of misassemblies and contig breaks in recent long-read genome assemblies, irrespective of the platform or assembly algorithm (Porubsky et al. 2019). To estimate the effect of SDs on the continuity of HiCanu assemblies, we aligned contigs from the CHM13 genome assemblies to the human reference genome (GRCh38) and tested if the ends of contigs mapped within SDs. Compared with the Canu, Peregrine, or ONT assemblies, HiCanu had the fewest contig breaks within SDs $(n=95)$ and the smallest overall fraction of contig ends mapping to SDs (49\%) (Table 4). Of these 95 regions, 59 (62\%) correspond to the longest $(>10 \mathrm{kbp}$ ) and most identical (>98\%) copy-number polymorphic duplicated regions of the human genome (Supplemental Fig. S11). These results indicate that SDs are better resolved using HiCanu; however, SDs still contribute disproportionately to the overall number of assembly breaks.

The defensin beta gene family, a set of copy-number variable genes (e.g., DEFB136, DEFB135, etc.) mapping to two locations on Chromosome 8p23.1 (which we refer to as the defensin beta cluster), is a case in point. This $\sim 6-\mathrm{Mbp}$ region plays an important role 


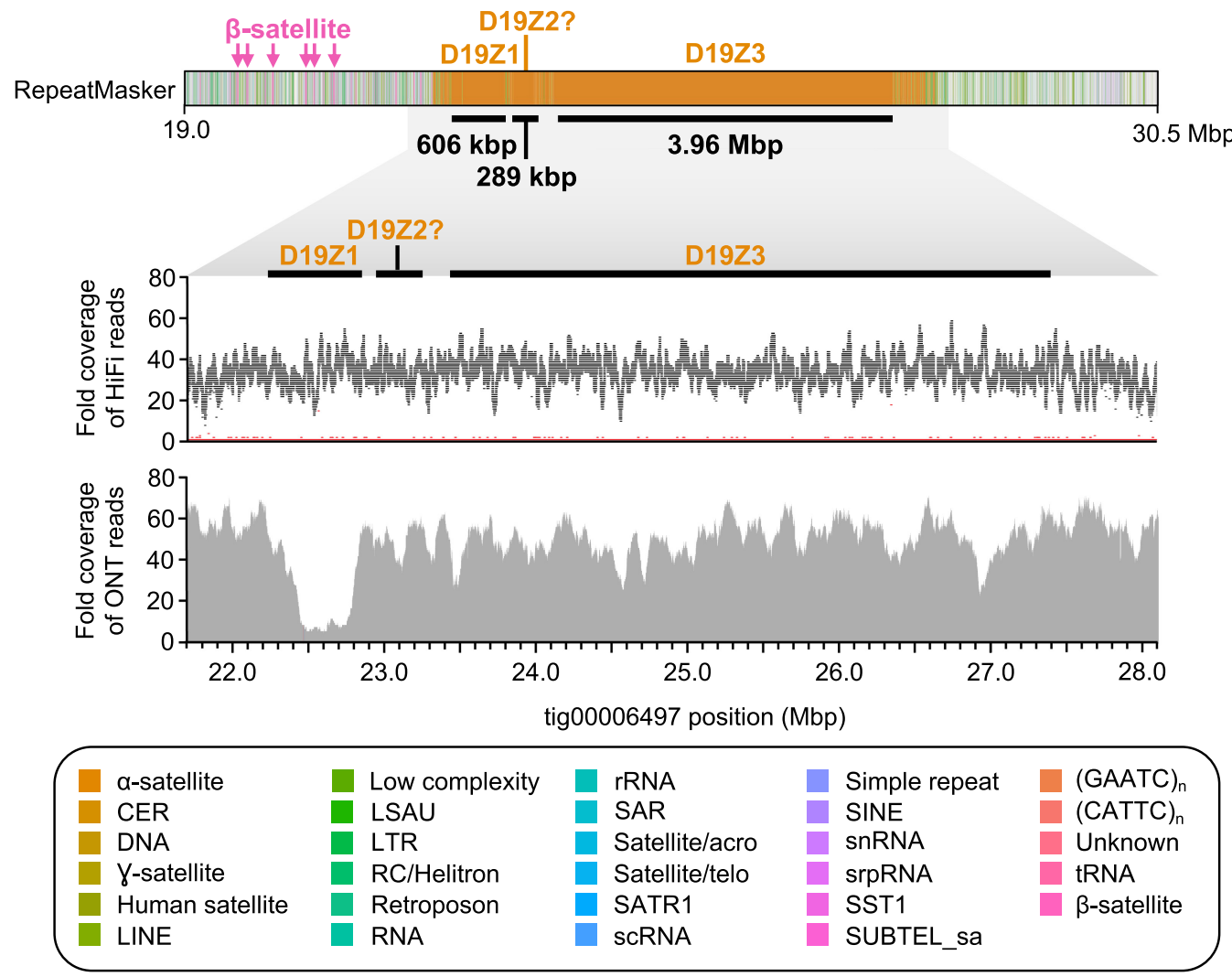

Figure 3. HiCanu assembly of the CHM13 Chromosome 19 centromere. RepeatMasker (Smit et al. 2013) of tig00006497 reveals three $\alpha$-satellite HOR arrays that reside within the Chromosome 19 centromere (D19Z1, D19Z2?, and D19Z3; marked with black bars). These HOR arrays are 606 kbp, 289 kbp, and $3.96 \mathrm{Mbp}$ in length, respectively, and are composed of a 13-mer, a complex higher-order HOR, and a dimeric HOR unit, respectively. The HOR repeat underlying D19Z2 shares limited sequence identity with the pG-A16 repeat previously described (Hulsebos et al. 1988; Choo et al. 1991; Finelli et al. 1996) and, therefore, is designated with a question mark. The $\alpha$-satellite HOR arrays have relatively uniform coverage of HiFi and ultralong Oxford Nanopore data, except for a drop in Oxford Nanopore sequencing coverage over the D19Z1 array, which may be owing to a misassembly, read mismapping, or biases in sequencing. The HiFi coverage plot shows fold coverage of the most common base (black) and the second most common base (red).

in immune function and disease (Weinberg et al. 2012; Mohajeri et al. 2016) and is known to be highly repetitive and difficult to assemble (Bakar et al. 2009). Previous reconstructions have relied on a BAC-by-BAC assembly approach (Mohajeri et al. 2016), and the first continuous assembly of this region in CHM13 was obtained via an independent approach using ultralong Nanopore data (GA Logsdon, MR Vollger, PH Hsieh, et al., in prep). Figure 4, A and $\mathrm{B}$ illustrates self-alignment dot plots at different stringencies of the defensin beta cluster from the T2T Chromosome $8 \mathrm{v} 3.0$ assembly. Figure $4 \mathrm{C}$ shows the de novo assembled contig alignment and correctness against this draft. Both the Canu and HiCanu assemblies of the HiFi data consist of four contigs without structural errors. In contrast, the complex inverted repeat structures resulted in misassembled and fragmented contigs in all other evaluated assemblies.

The rightmost contig breaks in the HiFi assemblies are likely owing to the presence of long, nearly identical repeats, which would require either longer reads or a careful examination of repeat copy number to resolve. We also investigated the fragmentation of HiCanu and Canu contigs at position $10.4 \mathrm{Mbp}$, which is not part of any observed repeat structure. Alignment of the raw HiFi reads onto this region with minimap2 (Li 2018) revealed the presence of a 450-bp region covered by only two HiFi reads (Supplemental Fig. S12), with a coverage drop present in both the 10- and 20kbp HiFi libraries. This coverage drop is flanked by a >250-bp sim- ple-sequence repeat (AAAGG). Suspecting a possible bias in the HiFi datatype, we further examined Chromosome $\mathrm{X}$, for which we have a complete CHM13 reference sequence available (Miga

Table 4. CHM13 contig ends found within SDs

\begin{tabular}{llccc}
\hline Genome & Assembly & $\begin{array}{c}\text { Total no. } \\
\text { of aligned } \\
\text { contig } \\
\text { ends }\end{array}$ & $\begin{array}{c}\text { Total no. } \\
\text { of contig } \\
\text { ends } \\
\text { within SDs }\end{array}$ & $\begin{array}{c}\text { Percentage of } \\
\text { contig ends } \\
\text { within SDs }\end{array}$ \\
\hline CHM13 & Canu ONT & 202 & 137 & $68 \%$ \\
& Canu & 322 & 170 & $53 \%$ \\
& Peregrine & 468 & 398 & $85 \%$ \\
HG002 & HiCanu & $\mathbf{1 9 2}$ & $\mathbf{9 5}$ & $\mathbf{4 9 \%}$ \\
& Canu ONT & 608 & 427 & $70 \%$ \\
& Canu & 1290 & 862 & $67 \%$ \\
& Peregrine & 862 & 741 & $86 \%$ \\
HG00733 & HiCanu & $\mathbf{4 1 8}$ & $\mathbf{2 3 4}$ & $\mathbf{5 6 \%}$ \\
& Canu ONT ${ }^{\mathrm{b}}$ & 606 & 492 & $81 \%$ \\
& Canu & 842 & 421 & $50 \%$ \\
& Peregrine & 874 & 716 & $82 \%$ \\
& HiCanu & $\mathbf{3 7 6}$ & $\mathbf{1 8 1}$ & $\mathbf{4 8 \%}$ \\
\hline
\end{tabular}

Previously published assemblies are indicated by superscripts: ${ }^{a}$ Miga et al. (2020), 'Shafin et al. (2020). All ONT assemblies were generated by Canu, as it achieved the highest NG50 and BAC resolution. The bestperforming result for each genome in each metric is highlighted in bold. 
A

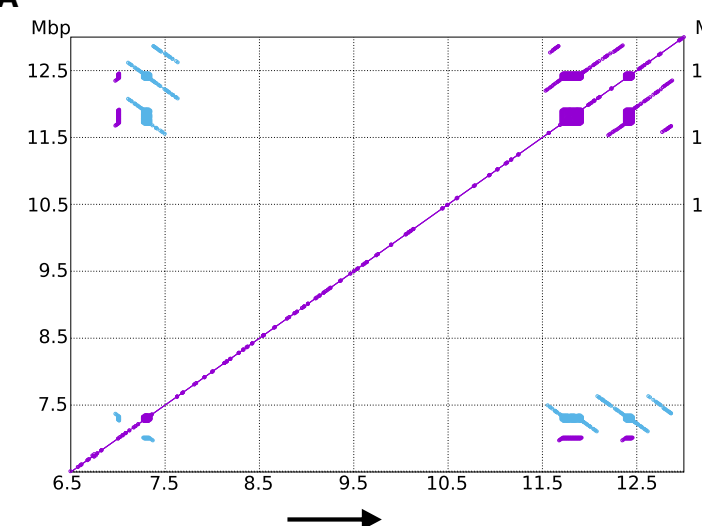

B

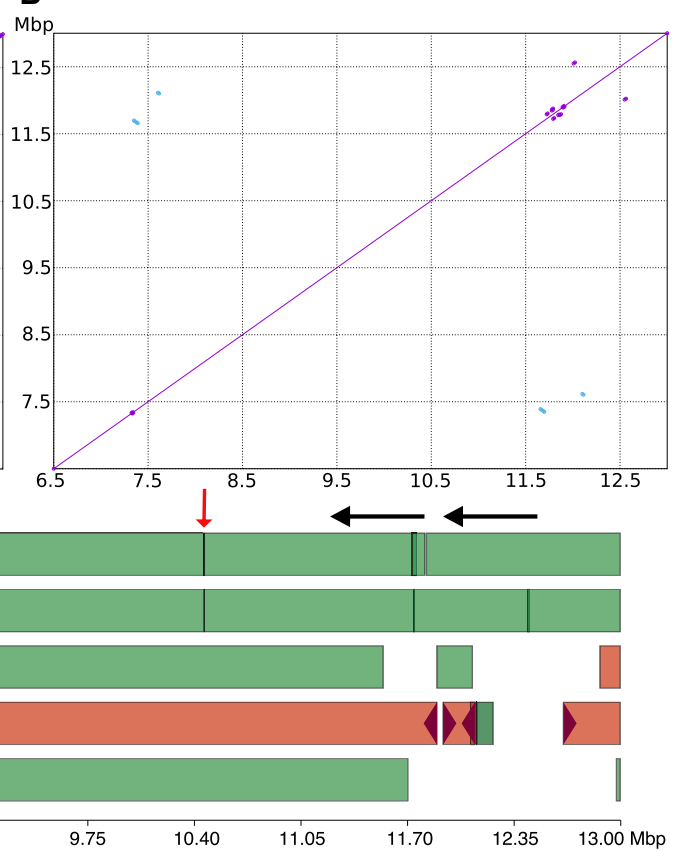

Figure 4. Chr 8 defensin beta cluster repeat structure and assembly comparison. (Top) NUCmer self-alignment dot plots (Kurtz et al. 2004) of the CHM13 reference defensin beta cluster at different alignment stringencies (Methods): $(A)>7$ kbp repeats at $98 \%$ identity. $(B)>7$ kbp repeats at $99.9 \%$ identity. Purple/blue indicates same/reverse strand matches. (C) Icarus (Mikheenko et al. 2016) visualization of contig alignments from both HiFi-based (Canu, HiCanu, Peregrine) and ultralong Nanopore-based assemblies (Canu ONT and Flye ONT) (Kolmogorov et al. 2019) produced by QUAST (Gurevich et al. 2013). White space in the alignment figure indicates the assembly was fragmented into short contigs ( $<50 \mathrm{kbp}$ ). Red color indicates misassembled contigs. The HiCanu assembly breaks at two of three SD instances that share high sequence similarity (black arrows) and at a region of systematic $\mathrm{HiFi}$ coverage depletion (red arrow).

et al. 2020). On this chromosome, we identified at least four additional cases of HiFi coverage dropout, with all four instances associated with long, low-complexity (A/G or T/C-rich) sequences. As our $\mathrm{HiFi}$ assembly of Chromosome $\mathrm{X}$ is split into just 13 large contigs (Supplemental Fig. S13), this coverage bias appears to be a current weakness of the HiFi chemistry.

\section{Discussion}

We have shown that HiCanu is capable of generating the most accurate and complete human genome assemblies to date and is able to achieve the resolution of repeats that are up to $99.99 \%$ identical. As a result, HiCanu surpasses prior HiFi and Nanopore ultra-long-read assemblies in terms of both repeat resolution and per-base consensus accuracy. HiFi data excels in resolving large highly-similar (but nonidentical) repeat instances. The remaining unresolved sequences seem to primarily represent satellite repeats (Supplemental Fig. S6). In particular, Figure 2 illustrates that HiCanu's reconstruction of human Chromosomes 1, 7, 9, and 16 notably improves continuity over the previous assembly of ultralong Nanopore reads (Koren et al. 2017; Kolmogorov et al. 2019; Shafin et al. 2020). These chromosomes contain several SDs exceeding $200 \mathrm{kbp}$ in length, requiring high-fidelity reads to identify variants and separate the individual copies. HiFi data further enabled draft assemblies of nine centromeric regions, which are one of the final challenges of automated telomere-to-telomere assembly. Assembly of other centromeric regions is likely limited by a low frequency of unique markers compared with current HiFi read lengths. In contrast, Chromosome $\mathrm{X}$ has the highest count of large (>20 kbp) near-identical (>99.9\%) repeats (Bailey et al 2002) that were better resolved by long, spanning Nanopore reads. Thus, the two technologies are complementary at present, and the best technology depends on the specific characteristics of the repeats and haplotypes being assembled.

HiCanu's approach to read correction permits considering only the highest identity overlaps during contig construction and is general enough to be applied to other applications such as metagenomic assembly. Although HiCanu is not as fast as some of the competing methods, we note that the number of CPU hours required for assembly of a human genome is under 4000, which could be completed on any modern cloud platform in less than a day for a few hundred dollars. This is 30 -fold less than recent Oxford Nanopore assemblies that required more than 100,000 CPU hours (Jain et al. 2018b; Shafin et al. 2020). At the time of writing, the most computationally expensive step of HiFi analysis is generating the data itself, because each individual HiFi read represents a consensus of multiple, aligned sequences of the same DNA molecule. Coupled with the instrument runtime and sequencing cost, HiCanu is a small fraction of the total project cost and duration (Supplemental Note 7). Most prior long-read assemblers have also required a final "polishing" step to improve consensus accuracy, which requires additional computation but can also introduce errors in repeat instances owing to ambiguous read mappings (Miga et al. 2020). Because of the initial accuracy of HiFi reads and because of the precise resolution of allelic variants and repeats, HiCanu assemblies do not require polishing.

When choosing HiFi, the library size should also be considered when beginning a sequencing project. Because HiFi read accuracy

\section{Genome Research}

www.genome.org 
depends on the size of the sequenced fragments (shorter equals more passes and higher accuracy), one should consider the relative importance of read length versus accuracy. A metagenomic project may aim for shorter, higher accuracy reads to confidently identify low-abundance strains, whereas a vertebrate genome project may benefit from longer reads to span midsized identical repeats. We also identified an apparent bias in the current HiFi chemistry at low-complexity A/G (T/C) repeats, leading to coverage drops and assembly fragmentation. This issue warrants further investigation and may limit the applicability of HiFi sequencing to genomes with large stretches of such repeats. Thus, identifying optimal sequencing strategies and developing methods that can combine multiple technologies remains an area for future research.

HiCanu's diploid assemblies accurately capture both alleles in long haplotype blocks of very high quality (QV50). In particular, HiCanu consistently recovered both haplotypes for the six canonical MHC typing genes in the human genome, improving upon recently developed HiFi-based methods for haplotype-resolved assembly (Garg et al. 2019; Porubsky et al. 2019). However, because HiFi data alone do not provide long-range linking evidence, HiCanu's contigs represent pseudohaplotypes that typically require additional information and processing to achieve chromosome-scale phasing. Canu also does not assign contigs to haplotypes and requires postprocessing with a tool such as Purge_dups (Guan et al. 2020) to split the diploid assembly into primary and alternate alleles. Although recent studies have successfully integrated HiFi data with additional long-range linkage information (Garg et al. 2019; Porubsky et al. 2019), we do not expect that significant improvements in phasing can be achieved by HiFi-only assemblies without an increase of HiFi read lengths. One option is postprocessing of HiCanu assemblies by a haplotypeaware scaffolder, such as FALCON-Phase (Kronenberg et al. 2019), which could potentially correct haplotype switch events and deliver further improvements to phasing accuracy and assembly continuity. In general, we feel that HiFi contigs combined with Hi-C phasing and scaffolding is a promising recipe for phased telomere-to-telomere vertebrate genome assembly, and we plan to integrate these data types in future versions of Canu.

\section{Methods}

\section{Mitigating errors in HiFi data}

Although HiFi reads are highly accurate compared with other longread sequencing technologies, they are not error free, which complicates the identification of reads originating from the same genomic loci during assembly. To identify and remove false read overlaps, we sought to increase the accuracy of HiFi data via read correction.

Wenger et al. (2019) observed that the majority of HiFi errors are in homopolymers, where the number of individually repeating nucleotides is incorrectly counted. To lessen the impact of such errors, HiCanu modifies the input reads by compressing every homopolymer to a single nucleotide. Our approach is similar to run-length encoding (RLE), which has been previously applied to 454 (Miller et al. 2008), PacBio CLR (Li 2016, 2018; Ruan and Li 2020), and Oxford Nanopore (Shafin et al. 2020) reads. However, HiCanu does not explicitly store the lengths of the compressed homopolymer stretches and instead reverts back to the uncompressed reads when needed.

Although the transition to homopolymer-compressed sequence space can reduce the specificity of the read alignment search, the corresponding reduction in the number of observed er- rors in the reads allow for a more restrictive alignment identity threshold (based on empirical analyses, we require a minimum overlap identity of 99\%). Subsequent steps are performed on the homopolymer-compressed sequences, whereas the detailed correspondence between positions of original and compressed versions is generated on the fly when necessary. Compressed reads are first subjected to overlap-based trimming (Koren et al. 2017). Although this step only affected one human assembly during development (HG002 sequel II system with pre-2.0 early access chemistry 15-kbp library available from https://github.com/humanpangenomics/HG002_Data_Freeze_v1.0) and does not appear to be necessary for newer HiFi data sets, we chose to enable it by default on all assemblies in this paper for consistency. This improvement suggests that a significant fraction of reads was structurally incorrect in the poorly performing library owing to a low-quality sequencing library. Because other libraries did not show this problem, it is likely future versions of the HiCanu pipeline can skip this step and reduce runtime by $>60 \%$.

To further reduce the influence of the errors in compressed HiFi reads, we have updated the OEA module of Canu (Holt et al. 2002; Koren et al. 2017). This module identifies errors in individual reads by jointly considering all of their overlapping reads. Every such read votes for the nucleotides at the positions that it covers based on the pairwise alignment of the overlapping regions. A read's position is considered erroneous if no other reads support the original sequence and the majority of votes agree on a particular change (by default $>50 \%$ and at least seven if there is a read supporting the original sequence). After the corrections are introduced, the alignment scores of the overlaps are recomputed, but the actual read sequences stored within the assembler are not modified as doing so would invalidate the previously computed overlap coordinates. Although our naive approach may not always be able to correct errors within highly-similar genomic repeats, such events are rare owing to the low number of errors in compressed HiFi reads and the high identity threshold used for gathering candidate overlaps.

Manual investigation of read alignments during HiCanu development revealed a previously unreported error mode in HiFi reads: incorrect repeat unit counts within microsatellite repeat arrays. Because the incorrect repeat counts are systematic and often supported by multiple reads, the conservative strategy described above is not able to correct them. Recognizing this, we modified the OEA procedure for recomputing overlap alignment scores to ignore sequence differences flanked by a microsatellite repeat in either read. Namely, the difference is ignored if five out of six nonoverlapping flanking $k$-mers are the same for any $k$ ranging between two and six on either side (starting at zero to $k-1 \mathrm{bp}$ from the difference). We note that this phenomenon deserves a deeper investigation, and our strategy can be improved to capture additional genomic differences, which are ignored by the current approach.

We evaluated the contribution of each of the above corrections using the recently completed CHM13 Chromosome X (Miga et al. 2020) as a reference. Raw, compressed, corrected, and masked 20-kbp HiFi reads were mapped and the mappings filtered to retain high-confidence alignments (Supplemental Note 1). Figure 1 shows the resulting alignment identity values, with each correction step boosting the identity of the aligned sequence. Each step (compression, correction, masking) contributes to this improvement (Supplemental Table S1; Supplemental Fig. S14). Although almost no (<1\%) raw HiFi reads map error free, $97.23 \%$ of the compressed, corrected, and masked reads map without a single difference. Without correction (compression + masking only), reads have similar median error to just compression, and less than half have perfect alignment. As we did not control for reads 
mapping from other chromosomes and as the Chromosome X sequence itself is not error free, this likely represents a lower bound on the percentage of error-free reads. To extend beyond Chr X, we also estimated read accuracy using $k$-mers from short-read data for all human genomes and found correction improved read accuracy across all data sets (Supplemental Note 1).

\section{Bogart modifications}

The Bogart module constructs a set of draft contigs from read overlap information. A detailed description is given by Koren et al. (2017). We describe here the modifications made for HiFi data.

\section{Overlap identity threshold}

Canu's initial overlap search uses a relaxed identity threshold to account for varying error rates between samples. Because overlap identities are changed by OEA and because we wished to avoid considering false-positive overlaps, Bogart first attempts to select a higher overlap identity threshold. Previously, Canu computed the identity of the best-scoring overlap on each side of every read (where score is defined as the number of matching bases in the overlap alignment) and set a threshold based on the median and MAD of the computed values (Koren et al. 2017). However, during the development, we realized that this way of computing the threshold was not informative for highly accurate reads because both the median and MAD were $100 \%$ across all tested data sets. Additionally, with the number of matching bases as a score, the read delivering the highest scoring overlap could come from a different haplotype in genomes with low heterozygosity. As a result, the selected threshold could inadvertently reflect the heterozygosity level of the organism rather than the accuracy of the reads. Based on empirical testing, we opted for an alternative two-step procedure. First, all overlaps with identity below the fixed value T (default $99.97 \%$ or three differences in $10,000 \mathrm{bp}$ ) are dropped. This step is aimed at removing from consideration the majority of the cross-haplotype overlaps even for low-heterozygosity organisms, for example, human heterozygosity rate of $0.1 \%$ (The 1000 Genomes Project Consortium 2012). Next, the identities of the highest scoring overlaps are collected as before, and the final threshold is set as the 90th\% percentile of this sample. It is possible that $99.97 \%$ is too stringent given higher error reads. We could detect this condition when the 90th percentile is too close to $99.97 \%$ and rerun the overlap filtering. However, on all data sets evaluated to date, the chosen identity threshold was $100 \%$. To support the desired overlap filtering stringency, the Canu codebase had to be modified to increase the precision with which the overlap identity values are stored.

\section{Handling heterozygous differences}

Bogart uses the filtered overlaps to identify and eliminate the reads likely representing sequencing artifacts and then constructs the best overlap graph (Miller et al. 2008), using the same overlap scoring function as before. This graph consists of the best-scoring overlap off both the $5^{\prime}$ and $3^{\prime}$ ends of each read, and the nonbranching paths within this graph form the preliminary layouts (arrangements of reads) that we refer to as greedy contigs. Bogart then inspects each greedy contig for long repeat instances that could have been incorrectly traversed. Repeats are detected by considering overlaps between the reads within and outside of the contig. If a suspected repeat has no reads spanning it or if there is a similarlength alternate read overlap, it is broken at the repeat boundary to avoid potential assembly errors as the method of Koren et al. (2017) to form the final draft contigs.
HiCanu aims to reconstruct long pseudohaplotype contigs (Vinson et al. 2005; Chin et al. 2016) - potentially switching between paternal and maternal alleles-and capture the alternative regions as shorter contigs. Unfortunately, the original Bogart approach described above led to the classification of extended homozygous regions within greedy contigs as unspanned repeats, leading to fragmentation of the pseudohaplotypes (Supplemental Fig. S15). In Canu, this behavior had been affecting only genomes with $>1 \%$ heterozygosity, because below this threshold most heterozygous differences were implicitly hidden by the relatively permissive threshold on overlap identity. With the high-accuracy HiFi data, and a correspondingly high overlap identity threshold, this overfragmentation became an issue even for human levels of heterozygosity.

In HiCanu, Bogart has an additional step to identify contigs representing alternative alleles within the set of greedy contigs, which we refer to as bubble contigs. As suggested by the name, the bubble contigs are related to the bubble subgraphs, typically considered by most assemblers. Candidate bubbles are found by identifying reads in each contig that have overlaps to some other, larger, contig. A read within a smaller contig can be placed in the larger contig if the overlaps between it and the reads in the larger contig are below a specified threshold of similar quality to the previously incorporated overlaps ( $0.1 \%$ by default). If the placements for both the first and last reads of a candidate contig are correctly oriented and placed at approximately the correct distance in the larger contig (75\%-125\% of the candidate contig size), the candidate contig is flagged as a bubble and its reads are excluded from later repeat detection. This avoids fragmentation of otherwise structurally correct pseudohaplotype contigs. Similar strategies have previously been used in short-read assembly (Pevzner et al. 2001; Zerbino and Birney 2008; Li et al. 2010; Gnerre et al. 2011), scaffolding metagenomes (Koren et al. 2011; Ghurye et al. 2019), and long-read assembly (Chin et al. 2016). Bubble contigs are also explicitly marked in the final output; however, because placements are not always found, especially for longer, more heterozygous alleles, we recommend using a postprocessing tool such as Purge_dups (Guan et al. 2020) to classify alternate alleles and remove any false duplications.

\section{Consensus calculation}

A consensus sequence is computed for all contigs using the uncompressed reads (trimmed to their good regions identified in compressed space). Canu originally used the layout produced by Bogart to estimate the position of each read within the contig and align it only to that location. Because the read layouts are now in homopolymer-compressed space, this strategy is unable to locate the read in uncompressed space. Instead, we compute the correspondence of each position in the compressed read to the original. This is used to update the read positions within the contig and expand the layout to uncompressed space. A modified version of the PBdagcon algorithm (Chin et al. 2013), with improved support for long contig sequences, is used to compute the final consensus sequence.

Currently, HiCanu will exclude erroneous reads from large contigs, but these erroneous reads may form their own short, lowcoverage contigs. This can slightly reduce average assembly accuracy for homozygous genomes versus a more permissive strategy like that in Canu. However, Canu's more permissive approach will incorrectly mix haplotypes and similar repeat copies. Further HiCanu consensus gains are possible with better handling of erroneous reads and a more sophisticated approach for predicting homopolymer run length, similar to MarginPolish (Shafin et al. 2020).

\section{Genome Research}

www.genome.org 


\section{Previously generated data and assemblies}

When available, previously published assemblies were downloaded and used. This included Oxford Nanopore UL Canu assemblies presented by Shafin et al. (2020) for HG0002 (80× Guppy HAC 2.3.5) and HG00733 (50× Guppy HAC 2.3.5), Canu + Racon assembly presented by Vollger et al. (2020), HG002 Canu assembly of $\mathrm{HiFi}$ reads presented by Wenger et al. (2019), Oxford Nanopore Canu assembly for CHM13 $(40 x+80 x$ UL Guppy HAC 3.1.5) presented by Miga et al. (2020), HiFi + Hi-C assemblies for HG002 presented by Garg et al. (2019), and HiFi+ Strand-seq assemblies for HG0733 presented by Porubsky et al. (2019). In the remaining cases, assemblies were run locally on the NIH Biowulf cluster.

The D. melanogaster HiFi data are available from NCBI BioProject database (https://www.ncbi.nlm.nih.gov/bioproject/) at PRJNA573706 (SRR10238607; median: 24.4 kbp; mean: 24.4 $\mathrm{kbp}$ ) and CLR (SRR9969843; median $13.3 \mathrm{kbp}$; mean: $17.2 \mathrm{kbp}$ ). Because of the high coverage, this data set was down-sampled to 40x HiFi data and 200x CLR data. These coverages represent $\sim 25 \%$ of the full run output. Because the exact parents of the F1 were not available, we used the previously generated short-read sequencing for binning and analysis (A4: SAMN00849823; ISO1: SRR6702604). The CHM13 Nanopore data are available at https:// s3.amazonaws.com/nanopore-human-wgs/chm13/nanopore/rel3/ rel3.fastq.gz and Illumina at GitHub (https://github.com/nano pore-wgs-consortium/CHM13\#10x-genomics-data). The HG002 Nanopore data are available at https://s3-us-west-2.amazonaws .com/human-pangenomics/index.html, HiFi at SRX5327410. HG002 and parent Illumina data are available from GIAB (Zook et al. 2016) at GitHub (https://github.com/genome-in-a-bottle/ giab_data_indexes), we only used the $2 \times 250$ data sets. The HG00733 Nanopore data are available at https://s3-us-west-2 .amazonaws.com/human-pangenomics/index.html, $\mathrm{HiFi}$ at ERX3831682. The Illumina data for HG00733 and parents were downloaded from the 1000 Genomes Project Consortium at https ://www.internationalgenome.org/data-portal/sample (The 1000 Genomes Project Consortium 2012). The CHM13 Chromosome 8 reference assembly is available at GitHub (https://github.com/ nanopore-wgs-consortium/CHM13\#downloads).

\section{Software commands}

HiCanu was run using Canu branch hicanu_rc with the following commands:

canu -assemble -p asm - d asm genomeSize=G -pacbiohifi reads. fastq.gz

with $\mathrm{G}=3.1 \mathrm{~g}$ for human and $150 \mathrm{~m}$ for $D$. melanogaster. This required $131 \mathrm{CPU}$ hours and $16 \mathrm{~GB}$ of memory for $D$. melanogaster, 2780 CPU hours and 66 GB of memory for the CHM13 10-kbp library, 5000 CPU hours and 119 GB of memory for the CHM13 20kbp library, 3999 CPU hours and 62 GB of memory for HG002, and $5233 \mathrm{CPU}$ hours and $50 \mathrm{~GB}$ of memory for HG00733.

For the standard Canu assembles, Canu branch hicanu_rc ran with the following command:

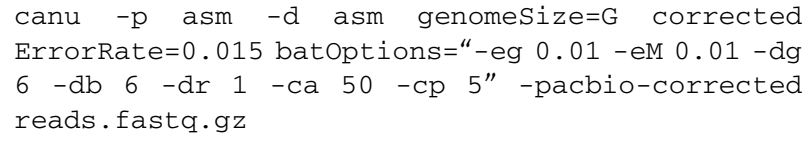

with $\mathrm{G}=3.1 \mathrm{~g}$ for human and $150 \mathrm{~m}$ for $D$. melanogaster. This required $232 \mathrm{CPU}$ hours and $12 \mathrm{~GB}$ of memory for D. melanogaster, 3524 CPU hours and 80 GB of memory for the CHM13 20-kbp library, and 3836 CPU hours and 47 GB of memory for HG00733.
For CLR data Canu branch hicanu_rc was run with the following command:

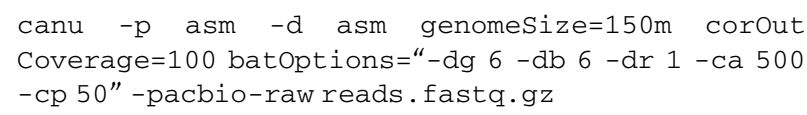

All HiFi assemblies required less than 12 wall-clock hours on the NIH Biowulf cluster quick partition with all jobs using $<120 \mathrm{~GB}$ RAM. We reran HG002 on our cluster limiting the maximum concurrent CPUs to 288, which required $30 \mathrm{~h}$. We estimated the cost of an AWS run using the c5d.18xlarge instance, which costs $\$ 3.456 /$ h. Assuming four reserved nodes (for a total of $72 \times 4=288$ CPUs) and an average runtime of $4200 \mathrm{CPU}$ hours with perfect parallelization, the run would complete in $14.5 \mathrm{~h}$. We increase this by a factor of 2.0 to account for any nonparallelized steps based on the experiments above for a cost of $\$ 3.456 \times 4 \times 29=\$ 401$. We note these estimates limited by differences in CPU and I/O between our cluster and AWS, as well as the overhead of waiting for a job to be scheduled on our cluster. The cost could also be reduced if additional nodes were spun up on-demand for the parallel portions of compute and spun down when not needed (as performed in Canu's DNAnexus implementation). We omit this from the estimate for simplicity. We also note that the assemblies could be completed faster if more nodes were allocated in parallel.

Peregrine assembler and SHIMMER ASMKit (0.1.5.3) was run with the command

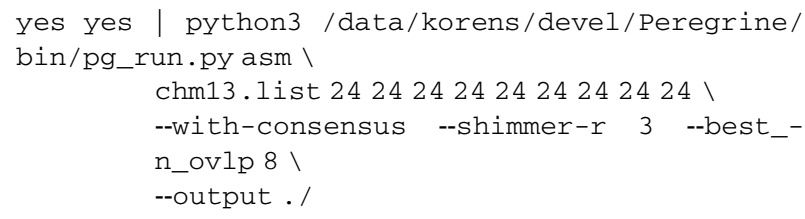

This required $7 \mathrm{CPU}$ hours and $29 \mathrm{~GB}$ of memory for D. melanogaster, $32 \mathrm{CPU}$ hours and $347 \mathrm{~GB}$ of memory for the CHM13 10-kbp library, $58 \mathrm{CPU}$ hours and $449 \mathrm{~GB}$ of memory for the CHM13 20-kbp library, 55 CPU hours and 407 GB for HG0002, and 63 CPU hours and 477 GB for HG00733.

\section{Commands for defensin beta cluster and Chr $X$ validation}

HiCanu contigs flagged as bubbles were excluded from the analysis. MUMmer (Kurtz et al. 2004) 3.23 was used to identify repeats with the command:

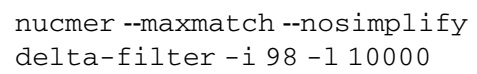

and high-stringency repeats

nucmer --maxmatch --noextend --nosimplify -1 500 -c 1000

delta-filter -i 99.9-1 10000

QUAST alignments were generated as

quast.py -t 20 --large --skip-unaligned-mis-contigs --min-alignment 10000 --min-identity 98.0 --extensive-mis-size 5000 --min-contig 50000

Icarus was patched not to show breaks at "small indels" and "stretches of mismatches," and used to visualize the resulting alignments.

\section{Commands for RepeatMasker}

RepeatMasker version 4.1.0 was run with the commands 
RepeatMasker -pa 8 -q -species=mammal $-x m$-dir $=-$ asm. out asm. fasta

on each contig $\geq 50 \mathrm{kbp}$ in the assembly. Centromeric arrays were identified by taking all hits marked as Satellite/centr and merging any hits within $100 \mathrm{bp}$ of each other using BEDTools (Quinlan and Hall 2010). Resulting arrays $>800 \mathrm{kbp}$ were reported. There were nine internal arrays whose start and end coordinates were at least $500 \mathrm{kbp}$ away from a contig end. These initial coordinates were manually curated based on reference alignments and are reported in Supplemental Table S12.

\section{Commands for MHC typing}

HLA*LA version commit 24930adadb0d2b6bcd69a271401dfc88a5d09f4d was run with the commands

HLA-ASM.pl --use_minimap2 1 --assembly_fasta \$asm -sampleID \$prefix --workingDir 'pwd'/\$prefix --truth reference_HLA_ASM/\$truth

where \$asm was the assembly, \$prefix was a unique identifier, and \$truth was either truth_HG002.txt or truth_HG00733.txt.

\section{Commands for Purge_dups}

Purge_dups version commit 8f580b41e6aa20c99383d6ff19b8689e93d7490e was run with the commands

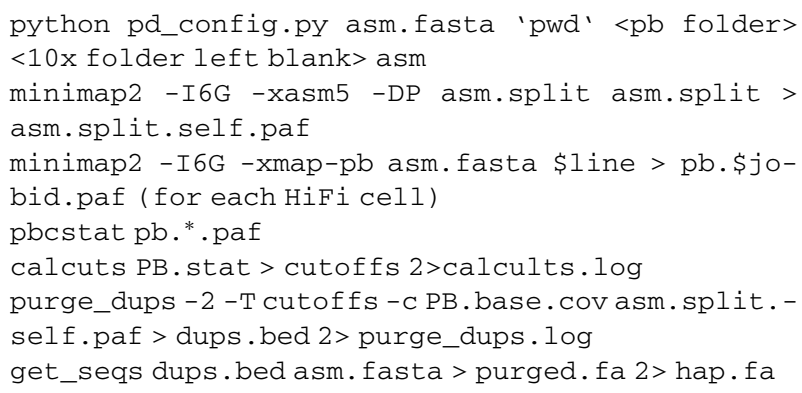

For D. melanogaster, an incorrect threshold was computed for the cutoffs owing to the entire genome being separated and so the cutoffs were manually adjusted to be

$$
50 \quad 1111522200 .
$$

The purged.fa output was then used as the primary set reported in the tables. To obtain the alternate set, we ran a second round of Purge_dups using hap.fa as the input assembly instead. This required an average of $20 \mathrm{CPU}$ hours and $7 \mathrm{MB}$ of memory for D. melanogaster, $59 \mathrm{CPU}$ hours and $24 \mathrm{MB}$ of memory for HG0002, and $74 \mathrm{CPU}$ hours and $24 \mathrm{MB}$ of memory for HG00733.

\section{Commands for Merqury}

Merqury version commit 154610d19ee6f4fead77da077af1ed7abdbe8866 was used. For each assembly and read set, canonical $k$-mers were built using meryl available as a binary within Canu:

meryl count $\mathrm{k}=<k$-size $>\quad<$ reads.fastq.gz $>$ output $<$ genome $>$. $k<k$-size $>$.meryl

meryl count $\mathrm{k}=<\mathrm{k}$-size $><$ asm.fasta $>$ output $<$ asm $>$. $k<$ size>.meryl

using $k=21$ for humans and $k=18$ for $D$. melanogaster based on (Fofanov et al. 2004). QV and $k$-mer completeness were obtained with

eval/spectra_cn.sh which converts $k$-mer Jaccard to distance as previously described (Ondov et al. 2019) and to a Phred score (Ewing and Green 1998). Haplotype blocks were estimated by first building parent-specific $k$-mer databases. $K$-mers in each parental data set were counted as above, then subtracted to obtain parent-specific $k$-mers, and finally intersected with the child (in the case of human data sets in which child Illumina data was available) with

$$
\begin{aligned}
& \text { trio/hapmers.sh } \\
& \text { trio/phased_block.sh }
\end{aligned}
$$

For further information see Supplemental Note 2 and https:// github.com/marbl/merqury/wiki.

\section{Commands used for QUAST}

QUAST 5.0.2 ran with the command

$$
\begin{aligned}
& \text { quast.py <asm> -o quast_results /<asm> -r <refer- } \\
& \text { ence> -t } 16 \text {-s --large }
\end{aligned}
$$

Variants were filtered using the pipeline from (Shafin et al. 2020) to filter errors in varying sites, including known SVs (HG002 only available from GIAB) (Zook et al. 2020) at ftp://ftp-trace.ncbi.nlm.nih.gov/giab/ftp/data/AshkenazimTrio/analysis/

NIST_SVs_Integration_v0.6/

HG002_SVs_Tier1plusTier2_v0.6.1.bed):

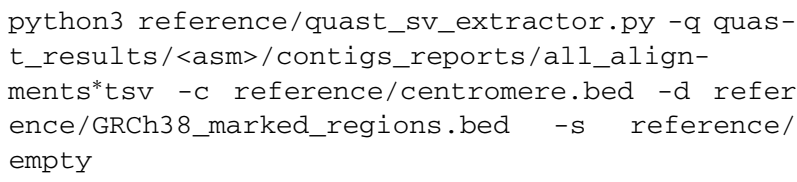

and

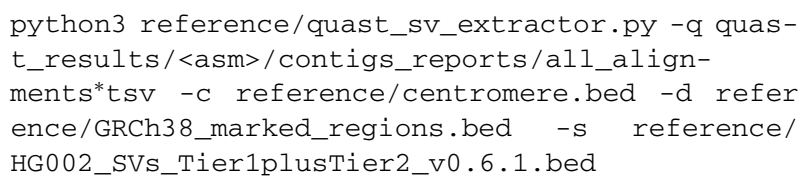

for HG002. We used https://www.ncbi.nlm.nih.gov/assembly/ GCF_000001215.4 filtered to remove any unassigned sequences for D. melanogaster (Chr 2L, Chr 2R, Chr 3L, Chr 3R, Chr 4, Chr M, Chr X, Chr Y only) and https://hgdownload.soe.ucsc.edu/ goldenPath/hg38/bigZips/hg38.fa.gz filtered to exclude alts and unaligned sequences (Chromosomes 1-22, X, Y, and MT only). Because no filtering file was available for D. melanogaster, we modified QUAST parameters to try to avoid false-positive misassembly counts with the command

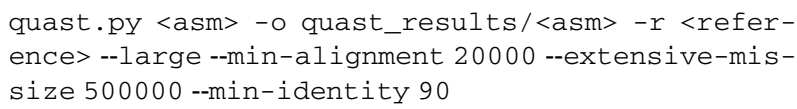

\section{Commands for BAC validation}

We used the BAC validation pipeline available at GitHub (at https ://github.com/skoren/bacValidation) run with default parameters. This pipeline aligns reads using minimap2 (Li 2018) and parses the SAM (Li et al. 2009) format to generate summary statistics. Output BAC identity was computed as the median across all BACs marked as correctly resolved. BAC libraries were downloaded from NCBI (CHM13: https://www.ncbi.nlm.nih.gov/nuccore/?term=VMRC 59+and+complete, HG00733: https:/www.ncbi.nlm.nih.gov/ nuccore/?term=VMRC62+and+complete). HiFi read alignments to the assembly and BAC sequences were visualized with the Integrative Genomics Viewer (IGV) (Robinson et al. 2011).

\section{Genome Research}

www.genome.org 


\section{Commands for variant analysis}

We downloaded trio-phased GIAB (Zook et al. 2019) variant calls for HG002 from ftp://ftp-trace.ncbi.nlm.nih.gov/Reference Samples/giab/release/AshkenazimTrio/HG002_NA24385_son/NI STv3.3.2/GRCh38/HG002_GRCh38_GIAB_highconf_CG-Illfb-Illse ntieonHC-Ion-10XsentieonHC-SOLIDgatkHC_CHROM1-22_v.3.3. 2_highconf_triophased.vcf.gz. We ran dipcall followed by vcfeval to estimate SNP sensitivity and precision (Supplemental Table S8) with the commands

run-dipcall hg002_purge GRCh38_full_analysis_ set_plus_decoy_hla.fa primary.fasta alts.fasta > hg002.mak

make -j1-f hg002. mak

\# exclude chrX/Y since there are no GIAB variants on them

gunzip -c hg002_purge.dip.vcf.gz |grep -v chrX | grep -v chry |bgzip -c > hg002_purge.dip_nohom. $\mathrm{vCf} \cdot \mathrm{gz}$

\# mark calls as homozygous alt in regions where only primary calls a variant and no alts map

gunzip -c hg002_purge.dip_nohom.vcf.gz | sed 's/ GAP2/./; / / /./1|1/;s/ID=\./ID=GAP2/' | grep -V 'HET $\backslash \mid$ GAP $\backslash \mid$ DIP' | bgzip -c > hg002_purge.dip.vCf.gz

tabix hg002_purge.dip_nohom.vcf.gz

tabixhg002_purge.dip.vcf.gz

\# measure statistics

rtg vcfeval -b HG002_GRCh38_GIAB_highconf_CGIllfb-IllsentieonHC-Ion-10XsentieonHC-

SOLIDgatkHC_CHROM1-22_v.3.3.2_highconf_triophased.vcf.gz -chg002_purge.dip.vcf.gz -e HG002_GRC h38_GIAB_highconf_CG-Illfb-IllsentieonHC-Ion10XsentieonHC-SOLIDgatkHC_CHROM1-

22_v.3.3.2_highconf_noinconsistent.bed -t GRCh38

_hs38d1.sdf -m annotate -o hom

To evaluate phasing, we evaluated the number of maternal and paternal variant calls out of the true positive calls in each contig and reported the total fraction of misphased variants (Supplemental Note 8).

\section{Commands for identifying contig ends}

Alignments were made between assemblies and GRCh38 using the following minimap2 command:

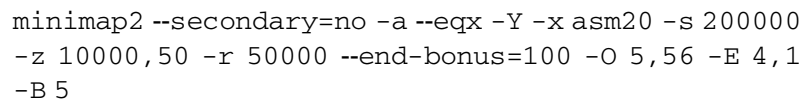

Contig ends that intersected SDs were identified by parsing the CIGAR string to find the location of contig ends and then by intersecting these locations with annotated SDs plus $10 \mathrm{kbp}$ on either side from the UCSC Genome Browser using the following commands:

bedtools slop -i \{segdups.bed\} -b $10000 \mid$ bedtools merge $-i->$ expanded. segdups.bed\} \&\& bedtools intersect $-\mathrm{a} \quad$ \{contig.ends.bed $-\mathrm{b}$ \{expanded. segdups.bed\}

\section{Data access}

All raw and processed sequencing data generated in this study have been submitted to the NCBI BioProject database (https ://www.ncbi.nlm.nih.gov/bioproject/) under accession number
PRJNA530776 (10 kbp: SRR9087597-SRR9087600; 20 kbp: SRR11292120-SRR11292123).

We have posted the down-sampled data sets, generated assemblies, and corrected CHM13 BAC sequences at https://obj .umiacs.umd.edu/marbl_publications/hicanu/index.html.

HiCanu is implemented within the Canu assembly framework and is available as Supplemental Code and from GitHub (https ://github.com/marbl/canu).

\section{Competing interest statement}

R.G. is an employee and shareholder of Pacific Biosciences. E.E.E. is on the scientific advisory board of DNAnexus. All other authors have no competing interests to declare.

\section{Acknowledgments}

This work was supported, in part, by the Intramural Research Program of the National Human Genome Research Institute, National Institutes of Health (S.N., B.P.W., A.R., A.M.P., and S.K.), as well as grants from the U.S. National Institutes of Health (NIH HG010169 and HG002385 to E.E.E.), National Human Genome Research Institute (NHGRI R21 1R21HG010548-01 to K.H.M., NHGRI U01 1U01HG010971 to K.H.M.), and National Institute of General Medical Sciences (NIGMS 1F32GM134558 to G.A.L.). E.E.E. is an investigator of the Howard Hughes Medical Institute. This work used the computational resources of the NIH HPC Biowulf cluster (https://hpc.nih.gov). We thank Pacific Biosciences for providing the D. melanogaster F1 and CHM13 20$\mathrm{kbp}$ data. We thank Heng Li and the other reviewers for their considered reviews.

\section{References}

The 1000 Genomes Project Consortium. 2012. An integrated map of genetic variation from 1092 human genomes. Nature 491: 56-65. doi:10.1038/ nature 11632

Bailey JA, Yavor AM, Massa HF, Trask BJ, Eichler EE. 2001. Segmental duplications: organization the current human genome project assembly. Genome Res and impact within 11: 1005-1017. doi:10.1101/gr.GR$1871 \mathrm{R}$

Bailey JA, Gu Z, Clark RA, Reinert K, Samonte RV, Schwartz S, Adams MD, Myers EW, Li PW, Eichler EE. 2002. Recent segmental duplications in the human genome. Science 297: 1003-1007. doi:10.1126/science .1072047

Bakar SA, Hollox EJ, Armour JAL. 2009. Allelic recombination between distinct genomic locations generates copy number diversity in human $\beta$ defensins. Proc Natl Acad Sci 106: 853-858. doi:10.1073/pnas .0809073106

Baldini A, Smith DI, Rocchi M, Miller OJ, Miller DA. 1989. A human alphoid DNA clone from the EcoRI dimeric family: genomic and internal organization and chromosomal assignment. Genomics 5: 822-828. doi:10 1016/0888-7543(89)90124-9

Berlin K, Koren S, Chin C-S, Drake JP, Landolin JM, Phillippy AM. 2015. Assembling large genomes with single-molecule sequencing and locality-sensitive hashing. Nat Biotechnol 33: 623-630. doi:10.1038/nbt.3238

Bickhart DM, Rosen BD, Koren S, Sayre BL, Hastie AR, Chan S, Lee J, Lam ET Liachko I, Sullivan ST, et al. 2017. Single-molecule sequencing and chromatin conformation capture enable de novo reference assembly of the domestic goat genome. Nat Genet 49: 643-650. doi:10.1038/ng.3802

Böhringer S, Gödde R, Böhringer D, Schulte T, Epplen JT. 2002. A software package for drawing ideograms automatically. Online J Bioinforma 1: 5161.

Chaisson MJP, Sanders AD, Zhao X, Malhotra A, Porubsky D, Rausch T, Gardner EJ, Rodriguez OL, Guo L, Collins RL, et al. 2019. Multi-platform discovery of haplotype-resolved structural variation in human genomes. Nat Commun 10: 1784. doi:10.1038/s41467-018-08148-z

Chin C-S, Khalak A. 2019. Human genome assembly in 100 minutes. bioRxiv doi:10.1101/705616

Chin C-S, Alexander DH, Marks P, Klammer AA, Drake J, Heiner C, Clum A, Copeland A, Huddleston J, Eichler EE, et al. 2013. Nonhybrid, finished 
microbial genome assemblies from long-read SMRT sequencing data. Nat Methods 10: 563-569. doi:10.1038/nmeth.2474

Chin C-S, Peluso P, Sedlazeck FJ, Nattestad M, Concepcion GT, Clum A, Dunn C, O'Malley R, Figueroa-Balderas R, Morales-Cruz A, et al. 2016. Phased diploid genome assembly with single-molecule real-time sequencing. Nat Methods 13: 1050-1054. doi:10.1038/nmeth.4035

Chin C-S, Wagner J, Zeng Q, Garrison E, Garg S, Fungtammasan A, Rautiainen M, Marschall T, Dilthey AT, Zook JM. 2019. A diploid assembly-based benchmark for variants in the major histocompatibility complex. bioRxiv doi:10.1101/831792

Choo KH, Vissel B, Nagy A, Earle E, Kalitsis P. 1991. A survey of the genomic distribution of $\alpha$ satellite DNA on all the human chromosomes, and derivation of a new consensus sequence. Nucleic Acids Res 19: 1179-1182. doi:10.1093/nar/19.6.1179

Church GM. 2005. The personal genome project. Mol Syst Biol 1: 2005.0030

Dilthey AT, Mentzer AJ, Carapito R, Cutland C, Cereb N, Madhi SA, Rhie A Koren S, Bahram S, McVean G, et al. 2019. HLA*LA-HLA typing from linearly projected graph alignments. Bioinformatics 35: 4394-4396. doi:10 .1093/bioinformatics/btz235

Ewing B, Green P. 1998. Base-calling of automated sequencer traces using phred. II: error probabilities. Genome Res 8: 186-194. doi:10.1101/gr.8 .3 .186

Finelli P, Antonacci R, Marzella R, Lonoce A, Archidiacono N, Rocchi M. 1996. Structural organization of multiple alphoid subsets coexisting on human Chromosomes 1, 4, 5, 7, 9, 15, 18, and 19. Genomics 38: 325-330. doi:10.1006/geno.1996.0635

Fofanov Y, Luo Y, Katili C, Wang J, Belosludtsev Y, Powdrill T, Belapurkar C, Fofanov V, Li TB, Chumakov S, et al. 2004. How independent are the appearances of n-mers in different genomes? Bioinformatics 20: $2421-$ 2428. doi:10.1093/bioinformatics/bth266

Garg S, Fungtammasan A, Carroll A, Chou M, Schmitt A, Zhou X, Mac S Peluso P, Hatas E, Ghurye J, et al. 2019. Efficient chromosome-scale haplotype-resolved assembly of human genomes. bioRxiv doi:10.1101/ 810341

Ghurye J, Treangen T, Fedarko M, Hervey WJ, Pop M. 2019. MetaCarvel: linking assembly graph motifs to biological variants. Genome Biol 20: 174. doi:10.1186/s13059-019-1791-3

Gnerre S, MacCallum I, Przybylski D, Ribeiro FJ, Burton JN, Walker BJ, Sharpe T, Hall G, Shea TP, Sykes S, et al. 2011. High-quality draft assemblies of mammalian genomes from massively parallel sequence data. Proc Natl Acad Sci 108: 1513-1518. doi:10.1073/pnas.1017351108

Gordon D, Huddleston J, Chaisson MJ, Hill CM, Kronenberg ZN, Munson KM, Malig M, Raja A, Fiddes I, Hillier LW, et al. 2016. Long-read sequence assembly of the gorilla genome. Science 352: aae0344. doi:10 .1126/science.aae0344

Guan D, McCarthy SA, Wood J, Howe K, Wang Y, Durbin R. 2020. Identifying and removing haplotypic duplication in primary genome assemblies. Bioinformatics. 36: 2896-2898. doi:10.1093/bioinfor matics/btaa025

Gurevich A, Saveliev V, Vyahhi N, Tesler G. 2013. QUAST: quality assessment tool for genome assemblies. Bioinformatics 29: 1072-1075. doi:10.1093/bioinformatics/btt086

Holt RA, Subramanian GM, Halpern A, Sutton GG, Charlab R, Nusskern DR, Wincker P, Clark AG, Ribeiro JC, Wides R, et al. 2002. The genome sequence of the malaria mosquito Anopheles gambiae. Science 298: 129149. doi:10.1126/science.1076181

Hoskins RA, Carlson JW, Wan KH, Park S, Mendez I, Galle SE, Booth BW, Pfeiffer BD, George RA, Svirskas R, et al. 2015. The release 6 reference sequence of the Drosophila melanogaster genome. Genome Res 25: 445-458. doi:10.1101/gr.185579.114

Hulsebos T, Schonk D, van Dalen I, Coerwinkel-Driessen M, Schepens J, Ropers HH, Wieringa B. 1988. Isolation and characterization of alphoid DNA sequences specific for the pericentric regions of chromosomes 4, 5 , 9, and 19. Cytogenet Genome Res 47: 144-148. doi:10.1159/000132533

Jain C, Koren S, Dilthey A, Phillippy AM, Aluru S. 2018a. A fast adaptive algorithm for computing whole-genome homology maps. Bioinformatics 34: i748-i756. doi:10.1093/bioinformatics/bty597

Jain M, Koren S, Miga KH, Quick J, Rand AC, Sasani TA, Tyson JR, Beggs AD, Dilthey AT, Fiddes IT, et al. 2018b. Nanopore sequencing and assembly of a human genome with ultra-long reads. Nat Biotechnol 36: 338-345. doi:10.1038/nbt.4060

Kolmogorov M, Yuan J, Lin Y, Pevzner PA. 2019. Assembly of long, errorprone reads using repeat graphs. Nat Biotechnol 37: 540-546. doi:10 1038/s41587-019-0072-8

Koren S, Phillippy AM. 2014. One chromosome, one contig: complete microbial genomes from long-read sequencing and assembly. Curr Opin Microbiol 23C: $110-120$.

Koren S, Treangen TJ, Pop M. 2011. Bambus 2: scaffolding metagenomes. Bioinformatics 27: 2964-2971. doi:10.1093/bioinformatics/btr520

Koren S, Harhay GP, Smith TP, Bono JL, Harhay DM, McVey SD, Radune D, Bergman NH, Phillippy AM. 2013. Reducing assembly complexity of microbial genomes with single-molecule sequencing. Genome Biol 14: R101. doi:10.1186/gb-2013-14-9-r101

Koren S, Walenz BP, Berlin K, Miller JR, Bergman NH, Phillippy AM. 2017. Canu: scalable and accurate long-read assembly via adaptive $k$-mer weighting and repeat separation. Genome Res 27: 722-736. doi:10 $.1101 /$ gr. 215087.116

Koren S, Rhie A, Walenz BP, Dilthey AT, Bickhart DM, Kingan SB, Hiendleder S, Williams JL, Smith TPL, Phillippy AM. 2018. De novo assembly of haplotype-resolved genomes with trio binning. Nat Biotechnol. 36: 1174-1182. doi:10.1038/nbt.4277

Kronenberg ZN, Fiddes IT, Gordon D, Murali S, Cantsilieris S, Meyerson OS, Underwood JG, Nelson BJ, Chaisson MJP, Dougherty ML, et al. 2018 High-resolution comparative analysis of great ape genomes. Science 360: eaar6343. doi:10.1126/science.aar6343

Kronenberg ZN, Rhie A, Koren S, Concepcion GT, Peluso P, Munson KM, Hiendleder S, Fedrigo O, Jarvis ED, Phillippy AM, et al. 2019 Extended haplotype phasing of de novo genome assemblies with FALCON-Phase. bioRxiv doi:10.1101/327064

Kurtz S, Phillippy A, Delcher AL, Smoot M, Shumway M, Antonescu C, Salzberg SL. 2004. Versatile and open software for comparing large genomes. Genome Biol 5: R12. doi:10.1186/gb-2004-5-2-r12

Li H. 2016. Minimap and miniasm: fast mapping and de novo assembly for noisy long sequences. Bioinformatics 32: 2103-2110. doi:10.1093/bioin formatics/btw152

Li H. 2018. Minimap2: pairwise alignment for nucleotide sequences Bioinformatics 34: 3094-3100. doi:10.1093/bioinformatics/bty191

Li H, Handsaker B, Wysoker A, Fennell T, Ruan J, Homer N, Marth G, Abecasis G, Durbin R, 1000 Genome Project Data Processing Subgroup. 2009. The Sequence Alignment/Map format and SAMtools. Bioinformatics 25: 2078-2079. doi:10.1093/bioinformatics/btp352

Li R, Zhu H, Ruan J, Qian W, Fang X, Shi Z, Li Y, Li S, Shan G, Kristiansen K, et al. 2010. De novo assembly of human genomes with massively parallel short read sequencing. Genome Res 20: 265-272. doi:10.1101/gr .097261 .109

McNulty SM, Sullivan BA. 2018. $\alpha$-Satellite DNA biology: finding function in the recesses of the genome. Chromosome Res 26: 115-138. doi:10 $1007 / \mathrm{s} 10577-018-9582-3$

Miga KH, Koren S, Rhie A, Vollger MR, Gershman A, Bzikadze A, Brooks S, Howe E, Porubsky D, Logsdon GA, et al. 2020. Telomere-to-telomere assembly of a complete human X chromosome. Nature doi:10.1038/ s41586-020-2547-7

Mikheenko A, Valin G, Prjibelski A, Saveliev V, Gurevich A. 2016. Icarus: visualizer for de novo assembly evaluation. Bioinformatics 32: 3321-3323. doi:10.1093/bioinformatics/btw379

Miller JR, Delcher AL, Koren S, Venter E, Walenz BP, Brownley A, Johnson J, Li K, Mobarry C, Sutton G. 2008. Aggressive assembly of pyrosequencing reads with mates. Bioinformatics 24: 2818-2824. doi:10.1093/bioin formatics/btn548

Miller JR, Koren S, Sutton G. 2010. Assembly algorithms for next-generation sequencing data. Genomics 95: 315-327. doi:10.1016/j.ygeno.2010.03 .001

Mohajeri K, Cantsilieris S, Huddleston J, Nelson BJ, Coe BP, Campbell CD, Baker C, Harshman L, Munson KM, Kronenberg ZN, et al. 2016. Interchromosomal core duplicons drive both evolutionary instability and disease susceptibility of the Chromosome 8p23.1 region. Genome Res 26: 1453-1467. doi:10.1101/gr.211284.116

Nagarajan N, Pop M. 2010. Sequencing and genome assembly using nextgeneration technologies. Methods Mol Biol Clifton NJ 673: $1-17$. doi:10 .1007/978-1-60761-842-3_1

Ondov BD, Starrett GJ, Sappington A, Kostic A, Koren S, Buck CB, Phillippy AM. 2019. Mash Screen: high-throughput sequence containment estimation for genome discovery. Genome Biol 20: 232. doi:10.1186/ s13059-019-1841-X

Pevzner PA, Tang H, Waterman MS. 2001. An Eulerian path approach to DNA fragment assembly. Proc Natl Acad Sci 98: 9748-9753. doi:10 $.1073 /$ pnas. 171285098

Pironon N, Puechberty J, Roizès G. 2010. Molecular and evolutionary characteristics of the fraction of human $\alpha$ satellite DNA associated with CENP-A at the centromeres of Chromosomes 1, 5, 19, and 21. BMC Genomics 11: 195 , doi:10.1186/1471-2164-11-195

Porubsky D, Ebert P, Audano PA, Vollger MR, Harvey WT, Munson KM, Sorensen M, Sulovari A, Haukness M, Ghareghani M, et al. 2019. A fully phased accurate assembly of an individual human genome. bioRxiv doi:10.1101/855049

Puechberty J, Laurent A-M, Gimenez S, Billault A, Brun-Laurent M-E, Calenda A, Marçais B, Prades C, Ioannou P, Yurov Y, et al. 1999. Genetic and physical analyses of the centromeric and pericentromeric regions of human Chromosome 5: recombination across 5cen. Genomics 56: 274-287. doi:10.1006/geno.1999.5742

\section{Genome Research}

www.genome.org 
Quinlan AR, Hall IM. 2010. BEDTools: a flexible suite of utilities for comparing genomic features. Bioinformatics 26: 841-842. doi:10.1093/bioinfor matics/btq033

Rhie A, Walenz BP, Koren S, Phillippy AM. 2020. Merqury: reference-free quality, completeness, and phasing assessment for genome assemblies. bioRxiv doi:10.1101/2020.03.15.992941

Robinson JT, Thorvaldsdóttir H, Winckler W, Guttman M, Lander ES, Getz G, Mesirov JP. 2011. Integrative genomics viewer. Nat Biotechnol 29: 24 26. doi:10.1038/nbt. 1754

Ruan J, Li H. 2020. Fast and accurate long-read assembly with wtdbg2. Nat Methods 17: 155-158. doi:10.1038/s41592-019-0669-3

Schneider VA, Graves-Lindsay T, Howe K, Bouk N, Chen HC, Kitts PA, Murphy TD, Pruitt KD, Thibaud-Nissen F, Albracht D, et al. 2017. Evaluation of GRCh38 and de novo haploid genome assemblies demonstrates the enduring quality of the reference assembly. Genome Res 27: 849-864. doi:10.1101/gr.213611.116

Schueler MG, Higgins AW, Rudd MK, Gustashaw K, Willard HF. 2001. Genomic and genetic definition of a functional human centromere. Science 294: 109-115. doi:10.1126/science.1065042

Seo JS, Rhie A, Kim J, Lee S, Sohn MH, Kim CU, Hastie A, Cao H, Yun JY, Kim J, et al. 2016. De novo assembly and phasing of a Korean human genome. Nature 538: 243-247. doi:10.1038/nature20098

Shafin K, Pesout T, Lorig-Roach R, Haukness M, Olsen HE, Bosworth C, Armstrong J, Tigyi K, Maurer N, Koren S, et al. 2020. Nanopore sequencing and the Shasta toolkit enable efficient de novo assembly of eleven human genomes. Nat Biotechnol doi:10.1038/s41587-020-0503-6

She X, Horvath JE, Jiang Z, Liu G, Furey TS, Christ L, Clark R, Graves T, Gulden CL, Alkan C, et al. 2004. The structure and evolution of centromeric transition regions within the human genome. Nature 430: 857864. doi:10.1038/nature02806

Smit A, Hubley R, Green P. 2013. RepeatMasker Open-4.0. http://www .repeatmasker.org.

Sullivan LL, Chew K, Sullivan BA. 2017. $\alpha$-Satellite DNA variation and function of the human centromere. Nucleus 8: 331-339. doi:10.1080/ 19491034.2017.1308989

Vinson JP, Jaffe DB, O'Neill K, Karlsson EK, Stange-Thomann N, Anderson S, Mesirov JP, Satoh N, Satou Y, Nusbaum C, et al. 2005. Assembly of polymorphic genomes: algorithms and application to Ciona savignyi. Genome Res 15: 1127-1135. doi:10.1101/gr.3722605

Vollger MR, Dishuck PC, Sorensen M, Welch AE, Dang V, Dougherty ML, Graves-Lindsay TA, Wilson RK, Chaisson MJP, Eichler EE. 2019. Longread sequence and assembly of segmental duplications. Nat Methods 16: $88-94$. doi:10.1038/s41592-018-0236-3
Vollger MR, Logsdon GA, Audano PA, Sulovari A, Porubsky D, Peluso P, Wenger AM, Concepcion GT, Kronenberg ZN, Munson KM, et al. 2020. Improved assembly and variant detection of a haploid human genome using single-molecule, high-fidelity long reads. Ann Hum Genet 84: $125-140$. doi:10.1111/ahg.12364

Vurture GW, Sedlazeck FJ, Nattestad M, Underwood CJ, Fang H, Gurtowski J, Schatz MC. 2017. GenomeScope: fast reference-free genome profiling from short reads. Bioinformatics 33: 2202-2204. doi:10.1093/bioinfor matics/btx153

Waterhouse RM, Seppey M, Simão FA, Manni M, Ioannidis P, Klioutchnikov G, Kriventseva EV, Zdobnov EM. 2018. BUSCO applications from quality assessments to gene prediction and phylogenomics. Mol Biol Evol 35: 543-548. doi:10.1093/molbev/msx319

Weinberg A, Jin G, Sieg S, McCormick T. 2012. The yin and yang of human $\beta$-defensins in health and disease. Front Immunol 3: 294. doi:10.3389/ fimmu.2012.00294

Wenger AM, Peluso P, Rowell WJ, Chang P-C, Hall RJ, Concepcion GT, Ebler J, Fungtammasan A, Kolesnikov A, Olson ND, et al. 2019. Accurate circular consensus long-read sequencing improves variant detection and assembly of a human genome. Nat Biotechnol 37: 1155-1162. doi:10 .1038/s41587-019-0217-9

Willard HF, Waye JS. 1987. Chromosome-specific subsets of human $\alpha$ satellite DNA: analysis of sequence divergence within and between chromosomal subsets and evidence for an ancestral pentameric repeat. J Mol Evol 25: 207-214. doi:10.1007/BF02100014

Zerbino DR, Birney E. 2008. Velvet: algorithms for de novo short read assembly using de Bruijn graphs. Genome Res 18: 821-829. doi:10.1101/ gr.074492.107

Zook JM, Catoe D, McDaniel J, Vang L, Spies N, Sidow A, Weng Z, Liu Y, Mason CE, Alexander N, et al. 2016. Extensive sequencing of seven human genomes to characterize benchmark reference materials. Sci Data 3 160025. doi:10.1038/sdata.2016.25

Zook JM, McDaniel J, Olson ND, Wagner J, Parikh H, Heaton H, Irvine SA, Trigg L, Truty R, McLean CY, et al. 2019. An open resource for accurately benchmarking small variant and reference calls. Nat Biotechnol 37: 561566. doi:10.1038/s41587-019-0074-6

Zook JM, Hansen NF, Olson ND, Chapman L, Mullikin JC, Xiao C, Sherry S, Koren S, Phillippy AM, Boutros PC, et al. 2020. A robust benchmark for detection of germline large deletions and insertions. Nat Biotechnol doi:10.1038/s41587-020-0538-8

Received March 14, 2020; accepted in revised form August 4, 2020. 


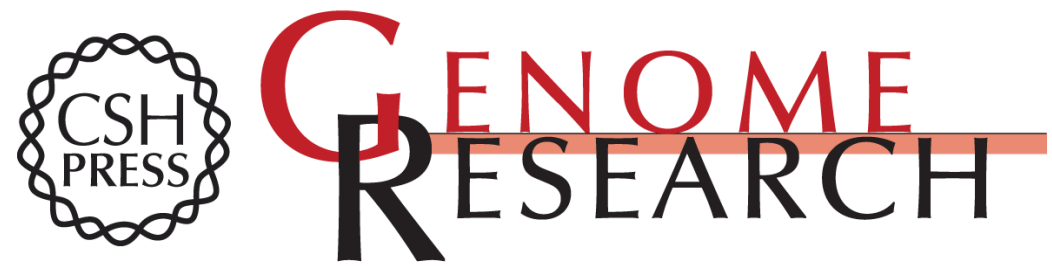

\section{HiCanu: accurate assembly of segmental duplications, satellites, and allelic variants from high-fidelity long reads}

Sergey Nurk, Brian P. Walenz, Arang Rhie, et al.

Genome Res. 2020 30: 1291-1305 originally published online August 14, 2020

Access the most recent version at doi:10.1101/gr.263566.120

Supplemental Material

References

Open Access

Creative Commons

License

Email Alerting Service
http://genome.cshlp.org/content/suppl/2020/09/02/gr.263566.120.DC1

This article cites 76 articles, 23 of which can be accessed free at: http://genome.cshlp.org/content/30/9/1291.full.html\#ref-list-1

Freely available online through the Genome Research Open Access option.

This article, published in Genome Research, is available under a Creative Commons License (Attribution 4.0 International), as described at http://creativecommons.org/licenses/by/4.0/.

Receive free email alerts when new articles cite this article - sign up in the box at the top right corner of the article or click here.

\section{Affordable, Accurate Sequencing.}

